\title{
A TRANSIENT EDDY CURRENT PROBLEM ON A MOVING DOMAIN. MATHEMATICAL ANALYSIS*
}

\author{
ALFREDO BERMÚDEZ ${ }^{\dagger}$, RAFAEL MUNOOZ-SOLA ${ }^{\dagger}$, CARLOS REALES $^{\ddagger}$, \\ RODOLFO RODRÍGUEZ ${ }^{\S}$, AND PILAR SALGADO ${ }^{\dagger}$
}

\begin{abstract}
The aim of this paper is to introduce and analyze a weak formulation of the transient eddy current problem governing electromagnetic forming in the axisymmetric case. The resulting problem is degenerate parabolic with the time derivative acting on a moving subdomain. Because of this, we have to resort to regularization arguments in order to prove its well-posedness. We also investigate additional regularity of the solution.
\end{abstract}

Key words. electromagnetic forming, transient eddy current problem, axisymmetric problem, degenerate parabolic problem, moving domain

AMS subject classifications. 35K20, 35K65

DOI. $10.1137 / 130914425$

1. Introduction. Electromagnetic forming (EMF) is a metal working process that relies on the use of electromagnetic forces to deform metallic workpieces at high speeds. A transient electric current is induced in a coil using a capacitor bank and high-speed switches. This current creates a magnetic field that penetrates the nearby conductive workpiece where an eddy current is generated. The magnetic field, together with the eddy current, produces Lorentz forces that drive the deformation of the workpiece [7, 13]. The workpiece can be reshaped without any contact from a tool, although in some instances the piece is pressed against a die or former. The technique is sometimes called high-velocity forming. The process works better with good electrical conductors such as copper or aluminum but it can also be adapted to work with poorer conductors such as steel.

In the thorough problem, the eddy current equations must be coupled with an adequate mechanical model for the deformation of the workpiece. In practice, this coupling can be achieved by means of a fixed-point iterative scheme. First, the transient electromagnetic field is determined with a fixed conducting workpiece at the initial position. Second, the Lorentz force computed from the resulting current density and magnetic induction is used as the driving force of the mechanical model, to

\footnotetext{
* Received by the editors March 26, 2013; accepted for publication (in revised form) October 4, 2013; published electronically December 3, 2013.

http://www.siam.org/journals/sima/45-6/91442.html

†Departamento de Matemática Aplicada, Universidade de Santiago de Compostela, Santiago de Compostela, 15706, Spain (alfredo.bermudez@usc.es, rafael.munoz@usc.es, mpilar.salgado@usc.es). The first and fifth authors were partially supported by Xunta de Galicia under research project INCITE09 $207047 \mathrm{PR}$ and by Ministerio de Ciencia e Innovación (Spain) under research projects Consolider MATHEMATICA CSD2006-00032 and MTM2008-02483. The second author was partially supported by Ministerio de Ciencia e Innovación (Spain) under research project MTM2009-07749 and by Xunta de Galicia under research project INCITE09 291083 PR.

‡Departamento de Matemáticas y Estadística, Universidad de Córdoba, Montería, Colombia (creales@correo.unicordoba.edu.co). This author was partially supported by a MECESUP fellowship (Chile).

${ }^{\S} \mathrm{CI}^{2} \mathrm{MA}$, Departamento de Ingeniería Matemática, Universidad de Concepción, Casilla 160-C Concepción, Chile (rodolfo@ing-mat.udec.cl). This author was partially supported by the BASAL project, CMM, Universidad de Chile (Chile), Anillo ANANUM, ACT1118, CONICYT (Chile), and Xunta de Galicia research project INCITE09 207047 PR.
} 
determine the position of the workpiece along the time. Then, the transient electromagnetic field with a moving conducting workpiece is recomputed. The last two steps are subsequently repeated until convergence.

In this paper we restrict our attention to the underlying electromagnetic model and take the motion of the workpiece as data. This motion introduces two difficulties to the eddy current problem. First, the conducting domain changes along time, because the workpiece changes its position. Also the velocity in the workpiece produces currents that in principle should be added in the Ohm's law. The difficulties arising from this additional term have been studied in [2] on a fixed domain. However, in EMF, the current density induced from the velocity terms is not significant, so that it is typically neglected.

The literature on mathematical analysis of electromagnetic problems on a moving domain is not abundant. As an example, let us mention [5], where the authors propose and analyze a sliding mesh-mortar method for a two-dimensional model of electric engines which takes into account the motion of the rotor. However, in this problem, the domain occupied by the moving part is always the same, which is not the case in the problem we are dealing with.

In the present paper we focus on problems with cylindrical symmetry, which allows stating the eddy current equations in terms of the azimuthal component of a magnetic vector potential defined in a meridian section of the domain (see, for instance, [1]). This leads to considering a transient problem where the term involving the time derivative appears only in a part of the domain which changes with time. Because of this, the classical theory for abstract nondegenerate parabolic problems (see, for instance, [6]) as well as that for degenerate parabolic problems on fixed domains (cf. [15]) do not seem to help for the mathematical analysis. This is why we resort to a regularization argument to prove well-posedness.

Since the ultimate goal of this research is to develop numerical tools for solving the transient problem, it is particularly interesting to study under which conditions the solution satisfies certain additional regularity, which may be necessary to guarantee the convergence of the numerical methods. With this end, first we prove time regularity without the need of any further assumptions on the data. Then, we determine conditions on the initial data which are necessary and sufficient for certain space regularity to hold. A relevant point in practice is that these conditions are typically satisfied in the EMF process.

The outline of this paper is as follows. In section 2, we describe the transient eddy current model and introduce a magnetic vector potential formulation under axisymmetric assumptions. In section 3 , we introduce a weak formulation and prove some preliminary results. Then, we prove that the resulting problem is well-posed in section 4 , and we prove some additional regularity results in section 5 . Finally, in an appendix, we give a sketch of the proof of a trace result on weighted Sobolev spaces.

2. Statement of the problem. We are interested in determining the electromagnetic field produced by a coil in a cylindrical workpiece; see Figure 2.1 (left) for an example. To ensure the cylindrical symmetry, we model the coil by several concentric rings with toroidal geometry, all carrying the same current intensity. On the other hand, to reduce the electromagnetic model to a bounded domain, we introduce a three-dimensional cylinder $\widetilde{\Omega}$ containing the coil and the workpiece with its boundary sufficiently far from them.

Because of the cylindrical symmetry, we are allowed to pose the problem in a meridian section of $\widetilde{\Omega}$, which we denote by $\Omega$. Notice that $\Omega$ is an open rectangle 

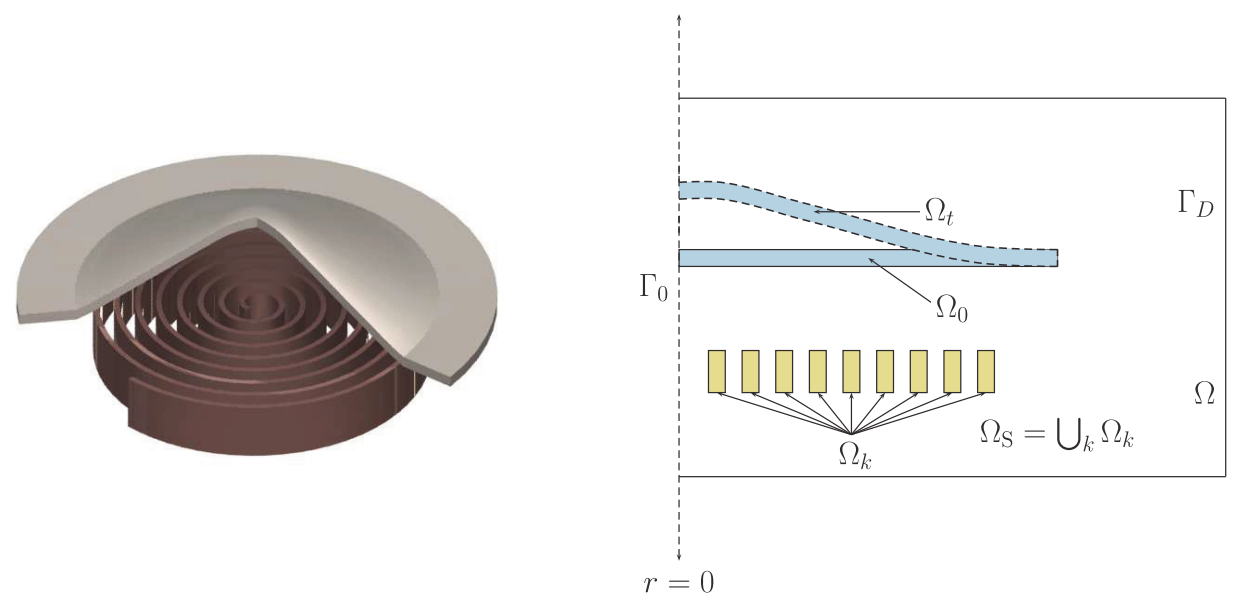

FIG. 2.1. Sketchs of the three-dimensional domain of an EMF system (left) and its meridian section (right).

contained in the half-plane $\{(r, z): r>0, z \in \mathbb{R}\}$ with an edge lying on the axis $r=0$, as shown in Figure 2.1 (right). We denote by $\Omega_{t}$ the meridian section of the workpiece at time $t$ and $\Omega_{\mathrm{S}}:=\Omega_{1} \cup \cdots \cup \Omega_{m}$, where $\Omega_{k}(k=1, \ldots, m)$ are the meridian sections of the turns of the coil. We assume that $\Omega_{t}$ and the sets $\Omega_{k}$ are open and that $\bar{\Omega}_{t} \cap \bar{\Omega}_{\mathrm{S}}=\emptyset$ for all $t$. Finally, we denote $\Gamma_{0}$ the intersection between $\partial \Omega$ and the axis $r=0$, and $\Gamma_{\mathrm{D}}:=\partial \Omega \backslash \Gamma_{0}$ (see Figure 2.1 (right), again).

We will use standard notation in electromagnetism:

- $\boldsymbol{E}$ is the electric field,

- $\boldsymbol{B}$ is the magnetic induction,

- $\boldsymbol{H}$ is the magnetic field,

- $\boldsymbol{J}$ is the current density,

- $\mu$ is the magnetic permeability,

- $\sigma$ is the electric conductivity.

The magnetic permeability $\mu$ is taken as a positive constant in the whole domain. The conductivity $\sigma$ vanishes outside the workpiece. This piece can be made of different materials, each with a different conductivity. We will make more precise assumptions on $\sigma$ below (cf. (3.2)-(3.3)).

In this kind of problem, the electric displacement can be neglected in Ampère's law, leading to the so called eddy current model:

$$
\begin{aligned}
\operatorname{curl} \boldsymbol{H} & =\boldsymbol{J}, \\
\frac{\partial \boldsymbol{B}}{\partial t}+\operatorname{curl} \boldsymbol{E} & =\mathbf{0}, \\
\operatorname{div} \boldsymbol{B} & =0 .
\end{aligned}
$$

This system must be completed with the relations

$$
\boldsymbol{B}=\mu \boldsymbol{H}
$$

and

$$
\boldsymbol{J}= \begin{cases}\sigma \boldsymbol{E} & \text { in the workpiece (unknown) } \\ \boldsymbol{J}_{\mathrm{S}} & \text { in the coil (data), } \\ \mathbf{0} & \text { in the air. }\end{cases}
$$


Notice that since the source current density $\boldsymbol{J}_{\mathrm{S}}$ is taken as given data, the conductivity $\sigma$ is taken as vanishing in the coil. The relation above can be written in a single equation as follows:

$$
\boldsymbol{J}=\sigma \boldsymbol{E}+\boldsymbol{J}_{\mathrm{S}},
$$

where $\boldsymbol{J}_{\mathrm{S}}$ is extended by zero outside the coil.

We assume that all the physical quantities are independent of the azimuthal coordinate $\theta$ and that the source current density field has only azimuthal nonzero component, i.e.,

$$
\boldsymbol{J}_{\mathrm{S}}(t, r, \theta, z)=J_{\mathrm{S}}(t, r, z) \boldsymbol{e}_{\theta}
$$

Then, proceeding as in [1] and [2], it can be shown that

$$
\begin{aligned}
\boldsymbol{H}(t, r, \theta, z) & =H_{r}(t, r, z) \boldsymbol{e}_{r}+H_{z}(t, r, z) \boldsymbol{e}_{z}, \\
\boldsymbol{B}(t, r, \theta, z) & =B_{r}(t, r, z) \boldsymbol{e}_{r}+B_{z}(t, r, z) \boldsymbol{e}_{z}, \\
\boldsymbol{E}(t, r, \theta, z) & =E(t, r, z) \boldsymbol{e}_{\theta}, \\
\boldsymbol{J}(t, r, \theta, z) & =J(t, r, z) \boldsymbol{e}_{\theta} .
\end{aligned}
$$

On the other hand, because of (2.3), we can introduce a magnetic vector potential $\boldsymbol{A}$ for $\boldsymbol{B}$ so that

$$
B=\operatorname{curl} A
$$

According to [2], this vector potential can be chosen of the form

$$
\boldsymbol{A}(t, r, \theta, z)=A(t, r, z) \boldsymbol{e}_{\theta}
$$

and such that (cf. (2.2))

$$
-\boldsymbol{E}=\frac{\partial \boldsymbol{A}}{\partial t}
$$

Therefore, from (2.1) and (2.4), the eddy current equations can be written in terms of this vector potential as follows:

$$
\operatorname{curl}\left(\frac{1}{\mu} \operatorname{curl} A\right)=J
$$

where

$$
\boldsymbol{J}=J \boldsymbol{e}_{\theta}, \quad \text { with } \quad J= \begin{cases}-\sigma \frac{\partial A}{\partial t} & \text { in } \Omega_{t}, \\ J_{\mathrm{S}} & \text { in } \Omega_{\mathrm{S}}(\text { data }), \\ 0 & \text { in } \Omega \backslash\left(\bar{\Omega}_{\mathrm{S}} \cup \bar{\Omega}_{t}\right) .\end{cases}
$$

Thus, we are led to the following parabolic-elliptic problem:

$$
\sigma \frac{\partial A}{\partial t} \boldsymbol{e}_{\theta}+\operatorname{curl}\left[\frac{1}{\mu} \operatorname{curl}\left(A \boldsymbol{e}_{\theta}\right)\right]=J_{\mathrm{S}} \boldsymbol{e}_{\theta} \quad \text { in }(0, T) \times \Omega .
$$

We complement this equation with homogeneous Dirichlet boundary conditions for $A$ on $\Gamma_{\mathrm{D}}$, which makes sense provided the boundary of $\widetilde{\Omega}$ is sufficiently far from the 
coil and the workpiece at all time $t \in[0, T]$. Moreover, as will be seen in the following section, a homogeneous Dirichlet boundary condition will be automatically imposed on $\Gamma_{0}$ from the fact that $\boldsymbol{A}=A \boldsymbol{e}_{\theta} \in H(\operatorname{curl}, \widetilde{\Omega}):=\left\{\boldsymbol{Z} \in L^{2}(\widetilde{\Omega})^{3}: \operatorname{curl} \boldsymbol{Z} \in L^{2}(\widetilde{\Omega})^{3}\right\}$.

3. Weak formulation and some preliminary results. The aim of this section is twofold: to introduce a weak formulation of problem (2.5) and to obtain some preliminary results which will be used in the next section to prove that the problem has a unique solution. With this end, first we introduce the functional framework we will use.

Let $L_{r}^{2}(\Omega)$ be the weighted Lebesgue space of all measurable functions $Z$ defined in $\Omega$ such that

$$
\|Z\|_{L_{r}^{2}(\Omega)}^{2}:=\int_{\Omega}|Z|^{2} r d r d z<\infty
$$

The weighted Sobolev space $H_{r}^{k}(\Omega)$ consists of all functions in $L_{r}^{2}(\Omega)$ whose derivatives up to order $k$ are also in $L_{r}^{2}(\Omega)$. We define the norms and seminorms of these spaces in the standard way. Let $L_{1 / r}^{2}(\Omega)$ be the space of all measurable functions $Z$ defined in $\Omega$ such that

$$
\|Z\|_{L_{1 / r}^{2}(\Omega)}^{2}:=\int_{\Omega} \frac{|Z|^{2}}{r} d r d z<\infty
$$

and let

$$
\widetilde{H}_{r}^{1}(\Omega):=H_{r}^{1}(\Omega) \cap L_{1 / r}^{2}(\Omega),
$$

endowed with its natural norm $\|Z\|_{\widetilde{H}_{r}^{1}(\Omega)}^{2}:=\|Z\|_{H_{r}^{1}(\Omega)}^{2}+\|Z\|_{L_{1 / r}^{2}(\Omega)}^{2}$. Since the curl of a vector field of the form $Z(r, z) \boldsymbol{e}_{\theta}$ reads

$$
\operatorname{curl}\left(Z \boldsymbol{e}_{\theta}\right)=-\frac{\partial Z}{\partial z} \boldsymbol{e}_{r}+\frac{1}{r} \frac{\partial(r Z)}{\partial r} \boldsymbol{e}_{z}
$$

it is well known that $Z \boldsymbol{e}_{\theta} \in H(\operatorname{curl}, \widetilde{\Omega})$ if and only if $Z \in \widetilde{H}_{r}^{1}(\Omega)$ (cf. [9]). Let

$$
\mathcal{V}:=\left\{Z \in \widetilde{H}_{r}^{1}(\Omega): Z=0 \text { on } \Gamma_{\mathrm{D}}\right\} .
$$

Let us emphasize that the functions in $\widetilde{H}_{r}^{1}(\Omega)$ have vanishing traces on $\Gamma_{0}$, so that those in $\mathcal{V}$ actually vanish on the whole boundary of $\Omega$.

Finally, following [9], we denote

$$
\|Z\|_{\widetilde{H}_{r}^{2}(\Omega)}^{2}:=\left|\frac{1}{r} \frac{\partial(r Z)}{\partial r}\right|_{H_{r}^{1}(\Omega)}^{2}+\left|\frac{\partial Z}{\partial z}\right|_{\widetilde{H}_{r}^{1}(\Omega)}^{2}+\|Z\|_{\widetilde{H}_{r}^{1}(\Omega)}^{2}
$$

and introduce the space $\widetilde{H}_{r}^{2}(\Omega):=\left\{Z \in \widetilde{H}_{r}^{1}(\Omega):\|Z\|_{\widetilde{H}_{r}^{2}(\Omega)}<\infty\right\}$.

Notation like $L_{r}^{2}(\cdot), \widetilde{H}_{r}^{1}(\cdot)$, etc., will also be used with analogous meaning for functions spaces on other subdomains.

Remark 3.1. The embedding

$$
\mathcal{V} \cap \widetilde{H}_{r}^{2}(\Omega) \subset H_{r}^{2}(\Omega)
$$




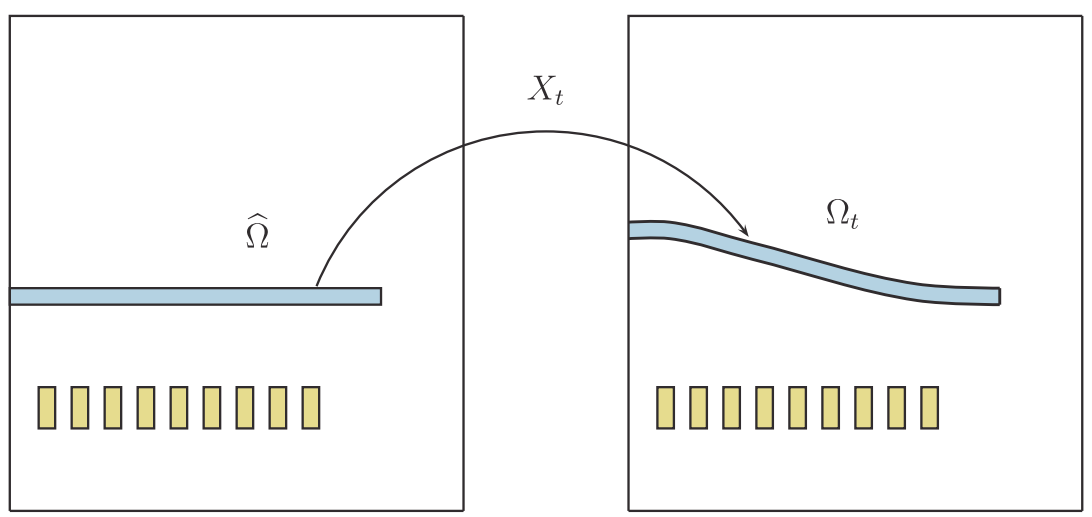

FIG. 3.1. Reference domain.

is an easy consequence of the results of [9]. In fact, let $Z \in \mathcal{V} \cap \widetilde{H}_{r}^{2}(\Omega)$ and set $f:=-\frac{\partial}{\partial r}\left[\frac{1}{r} \frac{\partial(r Z)}{\partial r}\right]-\frac{\partial^{2} Z}{\partial z^{2}} \in L_{r}^{2}(\Omega)$. Using the density in $\mathcal{V}$ of the space $\mathcal{D}(\Omega)$ of infinitely differentiable functions with compact support contained in $\Omega$ (which, in turn, follows by adapting the arguments from the proofs of [11, Theorem 4.3] and [9, Lemma 3.1]), we infer that $Z$ satisfies

$$
\int_{\Omega}\left[\frac{1}{r} \frac{\partial(r Z)}{\partial r} \frac{\partial(r V)}{\partial r}+\frac{\partial Z}{\partial z} \frac{\partial V}{\partial z} r\right] d r d z=\int_{\Omega} f V r d r d z \quad \forall V \in \mathcal{V} .
$$

Then, [9, Theorem 4.1] implies that $Z \in H_{r}^{2}(\Omega)$.

Since the domain $\Omega_{t}$ changes with time, we define a reference domain $\widehat{\Omega} \subset(0, \infty) \times$ $\mathbb{R}$ and an application $\boldsymbol{X}:[0, T] \times \overline{\widehat{\Omega}} \rightarrow \bar{\Omega}$ such that, for all $t \in[0, T]$,

$$
\begin{aligned}
\boldsymbol{X}_{t}: & \overline{\widehat{\Omega}} \longrightarrow \bar{\Omega}_{t}, \\
& \widehat{\boldsymbol{x}} \longmapsto \boldsymbol{X}(t, \widehat{\boldsymbol{x}})
\end{aligned}
$$

is a one-to-one correspondence which satisfies $\boldsymbol{X}_{t}(\widehat{\Omega})=\Omega_{t}$ (see Figure 3.1). For simplicity, we assume that $\boldsymbol{X}_{0}$ is the identity, so that $\widehat{\Omega}=\Omega_{0}$.

We also assume that $\boldsymbol{X}$ is sufficiently smooth with respect to space and time. More precisely, we make the following assumptions:

(i) forall $t \in[0, T], \quad \boldsymbol{X}_{t}: \bar{\Omega} \rightarrow \bar{\Omega}_{t}$ is a one-to-one correspondence and $\boldsymbol{X}_{t}(\widehat{\Omega})=$ $\Omega_{t}$

(ii) forall $t \in[0, T], \quad \boldsymbol{X}(t, \widehat{\boldsymbol{x}})$ lies on the axis $r=0$ if and only if $\widehat{\boldsymbol{x}}$ lies on the same axis;

(iii) the mapping $t \mapsto \boldsymbol{X}_{t}$ lies in $\mathcal{C}^{1}\left([0, T] ; \mathcal{C}^{1}(\overline{\widehat{\Omega}})^{2}\right)$;

(iv) $\operatorname{det}\left(D_{\hat{\boldsymbol{x}}} \boldsymbol{X}\right)(t, \widehat{\boldsymbol{x}})>0 \quad \forall(t, \widehat{\boldsymbol{x}}) \in[0, T] \times \overline{\widehat{\Omega}}$.

In assumption (iii) and thereafter, for any bounded open set $G \subset \mathbb{R}^{n}$, we denote by $\mathcal{C}^{1}(\bar{G})$ the set of functions in $\mathcal{C}(\bar{G}) \cap \mathcal{C}^{1}(G)$ such that all its first-order partial derivatives have continuous extensions to all of $\bar{G}$.

Assumptions (i) and (ii) are natural when $\boldsymbol{X}$ is the description in a meridian plane of a three-dimensional axisymmetric motion keeping invariant the azimuthal coordinate. Moreover, in such a case, assumptions (iii) and (iv) are satisfied provided the three-dimensional motion is sufficiently smooth. 
On the other hand, we impose the following geometric constraints on $\widehat{\Omega}$ :

(v) $\widehat{\Omega}$ is a Lipschitz bounded domain;

(vi) either $\overline{\widehat{\Omega}}$ does not intersect the axis $r=0$ or there exists $a>0$ such that the set $\{(r, z) \in \widehat{\Omega}: 0<r<a\}$ is a trapezoid with parallel sides aligned with the axis $r=0$;

(vii) $\overline{\widehat{\Omega}} \cap \bar{\Gamma}_{\mathrm{D}}=\emptyset$.

For further details and consequences of assumption (vi), see [11].

Finally, the conductivity $\sigma$ is taken such that

$$
\sigma(t, \boldsymbol{x})=\widehat{\sigma}(\widehat{\boldsymbol{x}}) \quad \text { with } \widehat{\boldsymbol{x}} \in \overline{\widehat{\Omega}}: \boldsymbol{x}=\boldsymbol{X}_{t}(\widehat{\boldsymbol{x}}),
$$

where $\widehat{\sigma}$ is the conductivity in the reference domain $\widehat{\Omega}$, which is a given measurable function satisfying

$$
0<\underline{\sigma} \leq \widehat{\sigma}(\widehat{\boldsymbol{x}}) \leq \bar{\sigma}, \quad \widehat{\boldsymbol{x}} \in \widehat{\Omega} .
$$

From a physical point of view, this means that the conductivity of each material point remains constant along the process.

Next, we introduce the noncylindrical open subset of $(0, T) \times \Omega$,

$$
Q:=\left\{(t, \boldsymbol{x}): \boldsymbol{x} \in \Omega_{t}, t \in(0, T)\right\},
$$

and, for $1 \leq p<\infty$, the Banach spaces of functions defined in $Q$,

$$
\begin{aligned}
L_{r}^{p}(Q) & :=\left\{\varphi: Q \rightarrow \mathbb{R} \text { measurable with } \int_{0}^{T} \int_{\Omega_{t}}|\varphi|^{p} r d r d z d t<\infty\right\}, \\
W_{r}^{1, p}(Q) & :=\left\{\varphi \in L_{r}^{p}(Q): \frac{\partial \varphi}{\partial t}, \frac{\partial \varphi}{\partial r}, \frac{\partial \varphi}{\partial z} \in L_{r}^{p}(Q)\right\},
\end{aligned}
$$

respectively endowed with their natural norms:

$$
\begin{aligned}
\|\varphi\|_{L_{r}^{p}(Q)}^{p} & :=\int_{0}^{T} \int_{\Omega_{t}}|\varphi|^{p} r d r d z d t, \\
\|\varphi\|_{W_{r}^{1, p}(Q)}^{p} & :=\|\varphi\|_{L_{r}^{p}(Q)}^{p}+\left\|\frac{\partial \varphi}{\partial t}\right\|_{L_{r}^{p}(Q)}^{p}+\left\|\frac{\partial \varphi}{\partial r}\right\|_{L_{r}^{p}(Q)}^{p}+\left\|\frac{\partial \varphi}{\partial z}\right\|_{L_{r}^{p}(Q)}^{p} .
\end{aligned}
$$

As usual, we denote $H_{r}^{1}(Q):=W_{r}^{1,2}(Q)$.

Now, we are in a position to write a weak formulation of (2.5). For this purpose, we multiply (2.5) by a test vector field $Z \boldsymbol{e}_{\theta}$ with $Z \in \mathcal{V}$, integrate in $\Omega$, and use a Green's formula to obtain

$$
\int_{\Omega_{t}} \sigma \frac{\partial A}{\partial t} Z r d r d z+a(A, Z)=\int_{\Omega_{\mathrm{S}}} J_{\mathrm{S}} Z r d r d z \quad \forall Z \in \mathcal{V}
$$

where

$$
a(A, Z):=\int_{\Omega} \frac{1}{\mu} \operatorname{curl}\left(A \boldsymbol{e}_{\theta}\right) \cdot \operatorname{curl}\left(Z \boldsymbol{e}_{\theta}\right) r d r d z .
$$


It is shown in $[9$, Propositions $2.1,3.1]$ that $a$ is a $\mathcal{V}$-elliptic bilinear form; namely, there exists $\alpha>0$ such that

$$
a(Z, Z) \geq \alpha\|Z\|_{\widetilde{H}_{r}^{1}(\Omega)}^{2} \quad \forall Z \in \mathcal{V} .
$$

Thus we are led to the following weak problem. (For the sake of notational compactness, here and hereafter $\partial_{t}$ will often be used to denote the derivative with respect to time.)

Problem 1. Given $J_{\mathrm{S}} \in L^{2}\left(0, T ; L_{r}^{2}\left(\Omega_{\mathrm{S}}\right)\right)$ and $A^{0} \in L_{r}^{2}\left(\Omega_{0}\right)$, find $A \in L^{2}(0, T ; \mathcal{V})$ with $\partial_{t} A \in L_{r}^{2}(Q)$ such that

$$
\begin{aligned}
\int_{\Omega_{t}} \sigma\left(\partial_{t} A\right) Z r d r d z+a(A, Z) & =\int_{\Omega_{\mathrm{S}}} J_{\mathrm{S}} Z r d r d z \quad \forall Z \in \mathcal{V} \quad \text { a.e. } t \in[0, T], \\
A(0) & =A^{0} \quad \text { in } \Omega_{0} .
\end{aligned}
$$

Remark 3.2. If $A \in L^{2}(0, T ; \mathcal{V})$ and $\partial_{t} A \in L_{r}^{2}(Q)$, it follows that $A \in H_{r}^{1}(Q)$. Hence, from the trace result proved in the appendix (cf. Lemma A.1), we have that $\left.A\right|_{\{0\} \times \Omega_{0}} \in L_{r}^{2}\left(\{0\} \times \Omega_{0}\right) \simeq L_{r}^{2}\left(\Omega_{0}\right)$. Thus, the initial condition in Problem 1 makes sense.

Notice that Problem 1 is degenerate parabolic, because the term including the time derivative of $A$ is only defined in the (moving) subdomain $\Omega_{t}$. Our first goal is to show that this degenerate problem is well-posed.

With this aim, we will use the Reynolds transport formula given in the next theorem, in which $\boldsymbol{v}=v_{r} \boldsymbol{e}_{r}+v_{z} \boldsymbol{e}_{z}$ is the velocity of the moving domain $\Omega_{t}$, which is defined for all $t \in[0, T]$ and $\boldsymbol{x} \in \bar{\Omega}_{t}$ by

$$
\boldsymbol{v}(t, \boldsymbol{x}):=\frac{\partial \boldsymbol{X}}{\partial t}(t, \widehat{\boldsymbol{x}}) \quad \text { with } \widehat{\boldsymbol{x}} \in \overline{\widehat{\Omega}}: \boldsymbol{x}=\boldsymbol{X}_{t}(\widehat{\boldsymbol{x}}) .
$$

Moreover, grad and div denote the differential operators applied to corresponding three-dimensional axisymmetric fields written in cylindrical coordinates, namely,

$$
\operatorname{grad} \phi:=\frac{\partial \phi}{\partial r} \boldsymbol{e}_{r}+\frac{\partial \phi}{\partial z} \boldsymbol{e}_{z} \quad \text { and } \quad \operatorname{div} \boldsymbol{v}:=\frac{1}{r} \frac{\partial\left(r v_{r}\right)}{\partial r}+\frac{\partial v_{z}}{\partial z}
$$

Let us remark that the Reynolds transport formula is an important analytical tool in continuum mechanics, whose proof, in the case of smooth motions and fields, can be found in many books (see, for instance, [10] or [8]). In what follows, we prove such a formula under weak smoothness assumptions, which will allow us to apply it to the analysis of Problem 1.

Theorem 3.1. Let $\boldsymbol{X}:[0, T] \times \overline{\widehat{\Omega}} \rightarrow \bar{\Omega}$ and $\boldsymbol{X}_{t}$ be defined as in (3.1). Suppose that assumptions (i)-(vi) hold true. Let $\boldsymbol{v}$ be given by (3.5) and $\sigma$ by (3.2). Then, the following Reynolds transport formula holds for all $\varphi, \psi \in H_{r}^{1}(Q)$ :

$$
\begin{aligned}
\frac{d}{d t} \int_{\Omega_{t}} \sigma \varphi \psi r d r d z= & \int_{\Omega_{t}} \sigma \frac{\partial(\varphi \psi)}{\partial t} r d r d z \\
& +\int_{\Omega_{t}} \sigma \varphi \psi \operatorname{div} \boldsymbol{v} r d r d z+\int_{\Omega_{t}} \sigma \operatorname{grad}(\varphi \psi) \cdot \boldsymbol{v} r d r d z
\end{aligned}
$$

in $\mathcal{D}^{\prime}((0, T))$ and a.e. in $[0, T]$. 
Before proving this theorem, we establish the following result, which will be used in its proof and also in what follows.

Lemma 3.2. Assumptions (i)-(vi) imply that $\operatorname{div} \boldsymbol{v}$ is bounded in $Q$.

Proof. First note that $\frac{\partial v_{r}}{\partial r}$ and $\frac{\partial v_{z}}{\partial z}$ are bounded in $Q$ (cf. assumptions (iii) and (iv)). If we denote by $(\widehat{r}, \widehat{z})$ a generic point in $\bar{\Omega}$, and by $(r, z)=\boldsymbol{X}(t, \widehat{r}, \widehat{z})$ the corresponding point in $\bar{\Omega}_{t}$, we have

$$
\frac{v_{r}(t, r, z)}{r}=\frac{\partial_{t} X_{1}(t, \widehat{r}, \widehat{z})}{X_{1}(t, \widehat{r}, \widehat{z})}
$$

where $X_{1}$ is just the first component of $\boldsymbol{X}$. We focus on the case $\overline{\widehat{\Omega}} \cap\{\widehat{r}=0\} \neq \emptyset$; otherwise, this quotient is bounded because of assumption (ii). Thus, it is enough to estimate the last quotient for $\widehat{r}>0$ small. With this aim, we first note that assumption (ii) implies that if $(0, \widehat{z}) \in \overline{\widehat{\Omega}}$, then $X_{1}(t, 0, \widehat{z})=0$ for all $t \in[0, T]$. Consequently, $\partial_{t} X_{1}(t, 0, \widehat{z})=0$ for $(t, 0, \widehat{z}) \in[0, T] \times \widehat{\widehat{\Omega}}$. Hence, using the mean value theorem and assumptions (iii) and (vi), we readily obtain that $\left|\partial_{t} X_{1}(t, \widehat{r}, \widehat{z})\right| \leq C \widehat{r}$ for $\widehat{r}>0$ small enough, $C$ being a suitable constant. This, together with (A.1) (cf. the proof of Lemma A.1, Step 3), yields the required boundedness of $\frac{\partial_{t} X_{1}(t, \widehat{r}, \vec{z})}{X_{1}(t, \widehat{r}, \vec{z})}$.

Proof of Theorem 3.1. Let $\varphi, \psi \in H_{r}^{1}(Q)$. Since $\mathcal{C}^{1}(\bar{Q})$ is dense in this space (cf. Step 5 from Lemma A.1), there exist $\varphi_{n}, \psi_{n} \in \mathcal{C}^{1}(\bar{Q}), n \in \mathbb{N}$, such that $\varphi_{n} \rightarrow \varphi$ and $\psi_{n} \rightarrow \psi$ in $H_{r}^{1}(Q)$. The proof of the classical Reynolds transport theorem (see, for instance, $[8$, Lemma 5.2]) can be readily adapted to cylindrical coordinates leading to (3.6) with $\varphi$ and $\psi$ substituted by $\varphi_{n}$ and $\psi_{n}$, respectively.

The next step is to pass to the limit in each of the four integrals of this expression in the sense of $L^{1}((0, T)$ ), taking advantage of the boundedness of $\sigma, \boldsymbol{v}$, and $\operatorname{div} \boldsymbol{v}$ (cf. Lemma 3.2 for the latter). For instance, for the last integral we have that

$$
\sigma \operatorname{grad}\left(\varphi_{n} \psi_{n}\right) \cdot \boldsymbol{v} \rightarrow \sigma \operatorname{grad}(\varphi \psi) \cdot \boldsymbol{v} \quad \text { in } L_{r}^{1}(Q)
$$

and, hence,

$$
\int_{\Omega_{t}} \sigma \operatorname{grad}\left(\varphi_{n} \psi_{n}\right) \cdot \boldsymbol{v} r d r d z \rightarrow \int_{\Omega_{t}} \sigma \operatorname{grad}(\varphi \psi) \cdot \boldsymbol{v} r d r d z \quad \text { in } L^{1}((0, T)) .
$$

Proceeding analogously with all the other integrals, we conclude that the function $t \in[0, T] \mapsto \int_{\Omega_{t}} \sigma \varphi \psi r d r d z$ lies in $W^{1,1}((0, T))$ and that (3.6) holds true. Thus, we conclude the proof.

Remark 3.3. It would be tempting to write (3.6) with the product $\varphi \psi$ replaced by $\phi \in W_{r}^{1,1}(Q)$. However, to prove such a result, we would need the density of $\mathcal{C}^{1}(\bar{Q})$ in this space and, to the best of the authors' knowledge, this has not been proved.

4. Well-posedness. The aim of this section is to prove that Problem 1 has a unique solution and to obtain a priori estimates.

In what follows, we will use the $t$-dependent bilinear form

$$
c(t, Y, Z):=-\int_{\Omega_{t}} \sigma Y Z \operatorname{div} \boldsymbol{v} r d r d z-\int_{\Omega_{t}} \sigma \operatorname{grad}(Y Z) \cdot \boldsymbol{v} r d r d z, \quad Y, Z \in \widetilde{H}_{r}^{1}(\Omega),
$$

which is related to the last two terms in the Reynolds transport formula from Theorem 3.1. The following Gårding-like inequality holds true. 
Lemma 4.1. There exists $\lambda>0$ such that

$$
a(Z, Z) \pm \frac{1}{2} c(t, Z, Z)+\lambda \int_{\Omega_{t}} \sigma|Z|^{2} r d r d z \geq \frac{\alpha}{2}\|Z\|_{\widetilde{H}_{r}^{1}(\Omega)}^{2},
$$

with $\alpha$ as in (3.4), for all $Z \in \widetilde{H}_{r}^{1}(\Omega)$ and all $t \in(0, T)$.

Proof. By using Young's inequality, we can write for any $\delta>0$

$$
\begin{aligned}
|c(t, Z, Z)| & =\left|\int_{\Omega_{t}} \sigma Z^{2} \operatorname{div} \boldsymbol{v} r d r d z+2 \int_{\Omega_{t}} \sigma Z \operatorname{grad} Z \cdot \boldsymbol{v} r d r d z\right| \\
& \leq\left(\|\operatorname{div} \boldsymbol{v}\|_{\infty}+\frac{\bar{\sigma}\|\boldsymbol{v}\|_{\infty}^{2}}{\delta}\right) \int_{\Omega_{t}} \sigma|Z|^{2} r d r d z+\delta\|Z\|_{H_{r}^{1}\left(\Omega_{t}\right)}^{2} .
\end{aligned}
$$

Thus, we conclude the proof from the ellipticity of $a$ (cf. (3.4)) by taking $\delta=\alpha$.

The next result is the first step to show that Problem 1 is well-posed. Here and hereafter, $C$ will denote a constant not necessarily the same at each occurrence but always independent of the data $J_{\mathrm{S}}$ and $A^{0}$ of Problem 1 .

THEOREM 4.2. If $J_{\mathrm{S}} \in H^{1}\left(0, T ; L_{r}^{2}\left(\Omega_{\mathrm{S}}\right)\right)$ and $A^{0} \in \widetilde{H}_{r}^{1}\left(\Omega_{0}\right)$, then there exists a solution to Problem 1 which satisfies $A \in L^{\infty}(0, T ; \mathcal{V})$ and

$$
\left\|\partial_{t} A\right\|_{L_{r}^{2}(Q)}+\|A\|_{L^{\infty}(0, T ; \mathcal{V})} \leq C\left[\left\|A^{0}\right\|_{\widetilde{H}_{r}^{1}\left(\Omega_{0}\right)}+\left\|J_{\mathrm{S}}\right\|_{H^{1}\left(0, T ; L_{r}^{2}\left(\Omega_{\mathrm{S}}\right)\right)}\right] .
$$

Proof. We proceed by Galerkin approximation and passing to the limit. Let $\left\{\phi_{n}\right\}_{n \in \mathbb{N}}$ be a "basis" of $\mathcal{V}$ in the following sense: $\left\{\phi_{n}\right\}_{n \in \mathbb{N}}$ is a set of linear independent elements of the space $\mathcal{V}$ whose linear span is dense in $\mathcal{V}$. Consider the family of finitedimensional subspaces $\mathcal{V}_{N}:=\left\langle\phi_{1}, \ldots, \phi_{N}\right\rangle$. The first step of the proof is to find a function of the form $A_{N}(t, r, z)=\sum_{j=1}^{N} A_{j N}(t) \phi_{j}(r, z)$ satisfying

$$
\begin{aligned}
& \int_{\Omega_{t}} \sigma\left(\partial_{t} A_{N}\right) \phi_{i} r d r d z+a\left(A_{N}, \phi_{i}\right) \\
& \quad=\int_{\Omega_{\mathrm{S}}} J_{\mathrm{S}} \phi_{i} r d r d z, \quad \text { a.e. } t \in[0, T], \quad 1 \leq i \leq N, \\
& \left.A_{N}(0)\right|_{\Omega_{0}}=\left.A_{N}^{0}\right|_{\Omega_{0}} .
\end{aligned}
$$

The initial condition $A_{N}^{0} \in \mathcal{V}_{N}$ must be chosen so that

$$
A_{N}^{0} \mid \Omega_{0} \stackrel{N}{\longrightarrow} A^{0} \quad \text { in } L_{r}^{2}\left(\Omega_{0}\right)
$$

and

$$
\left\|A_{N}^{0}\right\|_{\widetilde{H}_{r}^{1}(\Omega)} \leq C\left\|A^{0}\right\|_{\widetilde{H}_{r}^{1}\left(\Omega_{0}\right)} .
$$

To obtain $A_{N}^{0}$ we may proceed as follows. Let $\bar{A}^{0} \in \mathcal{V}$ be an extension of $A^{0}$ to $\Omega$ satisfying

$$
\left\|\bar{A}^{0}\right\|_{\widetilde{H}_{r}^{1}(\Omega)} \leq C\left\|A^{0}\right\|_{\widetilde{H}_{r}^{1}\left(\Omega_{0}\right)} .
$$

Such an $\bar{A}^{0}$ can be obtained, for instance, by means of a Nikolskii extension operator as in [11, Lemma 4.1] (here we use assumptions (v) and (vi)) and a smooth cut-off function vanishing in a neighborhood of $\bar{\Gamma}_{\mathrm{D}}$ and taking the value 1 in $\Omega_{0}$ (which exists 
because of assumption (vii)). Then, we take $A_{N}^{0} \in \mathcal{V}_{N}$ such that $\left\|A_{N}^{0}-\bar{A}^{0}\right\|_{\widetilde{H}_{r}^{1}(\Omega)} \stackrel{N}{\longrightarrow} 0$. This clearly implies (4.4) and together with (4.6) lead to (4.5). We write $A_{N}^{0}$ in terms of the basis $\left\{\phi_{1}, \ldots, \phi_{N}\right\}$ as

$$
A_{N}^{0}:=\sum_{j=1}^{N} b_{j N} \phi_{j} .
$$

Since problem (4.2)-(4.3) is degenerate, to prove the existence of a solution we introduce a parabolic regularization as follows: For small $\varepsilon>0$, we replace (4.2)-(4.3) by the "approximate" parabolic problem

$$
\begin{aligned}
& \int_{\Omega_{t}} \sigma\left(\partial_{t} A_{N}^{\varepsilon}\right) \phi_{i} r d r d z+\varepsilon \int_{\Omega}\left(\partial_{t} A_{N}^{\varepsilon}\right) \phi_{i} r d r d z+a\left(A_{N}^{\varepsilon}, \phi_{i}\right) \\
& \quad=\int_{\Omega_{\mathrm{S}}} J_{\mathrm{S}} \phi_{i} r d r d z \quad \text { a.e. } t \in[0, T], \quad i=1, \ldots, N, \\
& A_{N}^{\varepsilon}(0)=A_{N}^{0} \quad \text { in } \Omega .
\end{aligned}
$$

To obtain the matrix form of this problem, we write $A_{N}^{\varepsilon}(t)=\sum_{j=1}^{N} A_{j N}^{\varepsilon}(t) \phi_{j}$ and define $\boldsymbol{A}_{N}^{\varepsilon}(t):=\left(A_{j N}^{\varepsilon}(t)\right)_{1 \leq j \leq N}$,

$$
\begin{aligned}
\boldsymbol{F}_{N}(t) & :=\left(F_{i N}(t)\right)_{1 \leq i \leq N}, & F_{i N}(t) & :=\int_{\Omega_{\mathrm{S}}} J_{\mathrm{S}}(t) \phi_{i} r d r d z, \quad 1 \leq i \leq N, \\
\mathcal{K} & :=\left(\mathcal{K}_{i j}\right)_{1 \leq i, j \leq N}, & \mathcal{K}_{i, j} & :=a\left(\phi_{i}, \phi_{j}\right), \quad 1 \leq i, j \leq N, \\
\mathcal{M}(t) & :=\left(\mathcal{M}_{i j}(t)\right)_{1 \leq i, j \leq N}, & \mathcal{M}_{i, j}(t) & :=\int_{\Omega_{t}} \sigma \phi_{i} \phi_{j} r d r d z, \quad 1 \leq i, j \leq N, \\
\mathcal{N} & :=\left(\mathcal{N}_{i j}\right)_{1 \leq i, j \leq N}, & \mathcal{N}_{i, j} & :=\int_{\Omega} \phi_{i} \phi_{j} r d r d z, \quad 1 \leq i, j \leq N .
\end{aligned}
$$

Finally, let $\boldsymbol{b}_{N}:=\left(b_{j N}\right)_{1 \leq j \leq N}$ with $b_{j N}$ as in (4.7). Then, problem (4.8)-(4.9) reads as follows: Find $\boldsymbol{A}_{N}^{\varepsilon}:[0, T] \longrightarrow \mathbb{R}^{N}$ such that

$$
\begin{aligned}
{[\mathcal{M}(t)+\varepsilon \mathcal{N}] \partial_{t} \boldsymbol{A}_{N}^{\varepsilon}(t)+\mathcal{K} \boldsymbol{A}_{N}^{\varepsilon}(t) } & =\boldsymbol{F}_{N}(t), \\
\boldsymbol{A}_{N}^{\varepsilon}(0) & =\boldsymbol{b}_{N} .
\end{aligned}
$$

Since $\mathcal{M}(t)$ is symmetric positive semidefinite and $\mathcal{N}$ is symmetric positive definite, we have that $\mathcal{M}(t)+\varepsilon \mathcal{N}$ is invertible and this problem has a unique solution in $W^{1,1}\left(0, T ; \mathbb{R}^{N}\right)$. Furthermore, $\boldsymbol{A}_{N}^{\varepsilon} \in H^{1}\left(0, T ; \mathbb{R}^{N}\right)$, because

$$
\begin{aligned}
\left\|\partial_{t} \boldsymbol{A}_{N}^{\varepsilon}\right\|_{L^{2}\left(0, T ; \mathbb{R}^{N}\right)} & \leq \operatorname{ess~sup}_{0 \leq t \leq T}\left|[\boldsymbol{M}(t)+\varepsilon \mathcal{N}]^{-1}\right|\left[\left\|\boldsymbol{F}_{N}\right\|_{L^{2}\left(0, T ; \mathbb{R}^{N}\right)}+\left\|\mathcal{K} \boldsymbol{A}_{N}^{\varepsilon}\right\|_{L^{2}\left(0, T ; \mathbb{R}^{N}\right)}\right] \\
& \leq \frac{1}{\varepsilon}\left|\mathcal{N}^{-1}\right|\left[\left\|\boldsymbol{F}_{N}\right\|_{L^{2}\left(0, T ; \mathbb{R}^{N}\right)}+|\mathcal{K}|\left\|\boldsymbol{A}_{N}^{\varepsilon}\right\|_{L^{2}\left(0, T ; \mathbb{R}^{N}\right)}\right]<\infty
\end{aligned}
$$

where $|\cdot|$ denotes the matrix norm induced by the Euclidean norm in $\mathbb{R}^{N}$.

In order to pass to the limit as $\varepsilon$ goes to 0 , we need a priori estimates. With this end, we multiply (4.8) by $\partial_{t} A_{i N}^{\varepsilon}$ and sum up from $i=1$ to $N$ to obtain

$$
\int_{\Omega_{t}} \sigma\left|\partial_{t} A_{N}^{\varepsilon}\right|^{2} r d r d z+\varepsilon \int_{\Omega}\left|\partial_{t} A_{N}^{\varepsilon}\right|^{2} r d r d z+\frac{1}{2} \frac{d}{d t} a\left(A_{N}^{\varepsilon}, A_{N}^{\varepsilon}\right)=\int_{\Omega_{\mathrm{S}}} J_{\mathrm{S}}\left(\partial_{t} A_{N}^{\varepsilon}\right) r d r d z
$$


Integrating in time from 0 to $\tau(0<\tau \leq T)$ and using an integration by parts formula on the right-hand side, we derive

$$
\begin{aligned}
\int_{0}^{\tau} & \int_{\Omega_{t}} \sigma\left|\partial_{t} A_{N}^{\varepsilon}(t)\right|^{2} r d r d z d t+\varepsilon \int_{0}^{\tau} \int_{\Omega}\left|\partial_{t} A_{N}^{\varepsilon}(t)\right|^{2} r d r d z d t+\frac{1}{2} a\left(A_{N}^{\varepsilon}(\tau), A_{N}^{\varepsilon}(\tau)\right) \\
= & \frac{1}{2} a\left(A_{N}^{0}, A_{N}^{0}\right)+\int_{\Omega_{\mathrm{S}}} J_{\mathrm{S}}(\tau) A_{N}^{\varepsilon}(\tau) r d r d z-\int_{\Omega_{\mathrm{S}}} J_{\mathrm{S}}(0) A_{N}^{0} r d r d z \\
& \quad-\int_{0}^{\tau} \int_{\Omega_{\mathrm{S}}}\left[\partial_{t} J_{\mathrm{S}}(t)\right] A_{N}^{\varepsilon}(t) r d r d z d t
\end{aligned}
$$

Hence, the ellipticity of $a,(4.5)$, and Young's inequality lead to

$$
\begin{aligned}
& \int_{0}^{\tau} \int_{\Omega_{t}} \sigma\left|\partial_{t} A_{N}^{\varepsilon}(t)\right|^{2} r d r d z d t+\varepsilon \int_{0}^{\tau} \int_{\Omega}\left|\partial_{t} A_{N}^{\varepsilon}(t)\right|^{2} r d r d z d t+\frac{\alpha}{4}\left\|A_{N}^{\varepsilon}(\tau)\right\|_{\widetilde{H}_{r}^{1}(\Omega)}^{2} \\
& \quad \leq C\left[\left\|A^{0}\right\|_{\widetilde{H}_{r}^{1}\left(\Omega_{0}\right)}^{2}+\left\|J_{\mathrm{S}}\right\|_{H^{1}\left(0, T ; L_{r}^{2}\left(\Omega_{\mathrm{S}}\right)\right)}^{2}+\int_{0}^{\tau}\left\|A_{N}^{\varepsilon}(t)\right\|_{\widetilde{H}_{r}^{1}(\Omega)}^{2} d t\right],
\end{aligned}
$$

whence, by applying Gronwall's lemma (see, for instance, [12, Lemma 1.4.1]), it follows that

$$
\begin{aligned}
& \int_{0}^{\tau} \int_{\Omega_{t}} \sigma\left|\partial_{t} A_{N}^{\varepsilon}(t)\right|^{2} r d r d z d t+\varepsilon \int_{0}^{\tau} \int_{\Omega}\left|\partial_{t} A_{N}^{\varepsilon}(t)\right|^{2} r d r d z d t \\
& \quad+\frac{\alpha}{4}\left\|A_{N}^{\varepsilon}(\tau)\right\|_{\widetilde{H}_{r}^{1}(\Omega)}^{2} \leq C\left[\left\|A^{0}\right\|_{\widetilde{H}_{r}^{1}\left(\Omega_{0}\right)}^{2}+\left\|J_{\mathrm{S}}\right\|_{H^{1}\left(0, T ; L_{r}^{2}\left(\Omega_{\mathrm{S}}\right)\right)}^{2}\right] .
\end{aligned}
$$

Thus, we have proved the following a priori estimates:

- $\partial_{t} A_{N}^{\varepsilon}$ is bounded in $L_{r}^{2}(Q)$,

- $\sqrt{\varepsilon} \partial_{t} A_{N}^{\varepsilon}$ is bounded in $L^{2}\left(0, T ; L_{r}^{2}(\Omega)\right)$,

- $A_{N}^{\varepsilon}$ is bounded in $L^{\infty}(0, T ; \mathcal{V})$.

Therefore, for fixed $N$, there exists $A_{N} \in L^{\infty}(0, T ; \mathcal{V})$ with $\partial_{t} A_{N} \in L_{r}^{2}(Q)$ and a sequence $\left\{\varepsilon_{n}\right\}_{n \in \mathbb{N}}$ converging to 0 such that

- $\partial_{t} A_{N}^{\varepsilon_{n}} \rightarrow \partial_{t} A_{N}$ weakly in $L_{r}^{2}(Q)$,

- $\sqrt{\varepsilon_{n}} \partial_{t} A_{N}^{\varepsilon_{n}} \rightarrow 0$ weakly in $L^{2}\left(0, T ; L_{r}^{2}(\Omega)\right)$,

- $A_{N}^{\varepsilon_{n}} \rightarrow A_{N}$ weakly-star in $L^{\infty}(0, T ; \mathcal{V})$.

In particular, because of Lemma A.1, this yields $\left.A_{N}(0)\right|_{\Omega_{0}}=\lim _{n \rightarrow \infty}\left[\left.A_{N}^{\varepsilon_{n}}(0)\right|_{\Omega_{0}}\right]=$ $\left.A_{N}^{0}\right|_{\Omega_{0}}$, where the limit is weak in $L_{r}^{2}\left(\Omega_{0}\right)$, so that $A_{N}$ satisfies the initial condition (4.3). Moreover, one can multiply (4.8) by an arbitrary function in $\mathcal{D}((0, T))$, integrate in time, and pass to the limit for $\varepsilon=\varepsilon_{n} \rightarrow 0$ as $n \rightarrow \infty$. This allows us to show that $A_{N}$ satisfies (4.2), too.

Furthermore, it is also possible to pass to the limit in estimate (4.13) (see, for instance, $[4$, Propositions III.5, III.12]) to obtain

$$
\begin{aligned}
& \int_{0}^{\tau} \int_{\Omega_{t}} \sigma\left|\partial_{t} A_{N}(t)\right|^{2} r d r d z d t+\frac{\alpha}{4}\left\|A_{N}\right\|_{L^{\infty}(0, T ; \mathcal{V})}^{2} \\
& \quad \leq C\left[\left\|A^{0}\right\|_{\widetilde{H}_{r}^{1}\left(\Omega_{0}\right)}^{2}+\left\|J_{\mathrm{S}}\right\|_{H^{1}\left(0, T ; L_{r}^{2}\left(\Omega_{\mathrm{S}}\right)\right)}^{2}\right] \quad \text { a.e. } \tau \in[0, T] .
\end{aligned}
$$


The above estimate allows us to conclude that there exists $A \in L^{\infty}(0, T ; \mathcal{V})$ with $\partial_{t} A \in L_{r}^{2}(Q)$ and a subsequence of $\left\{A_{N}\right\}$, still denoted in the same way, such that

- $A_{N} \rightarrow A$ weakly-star in $L^{\infty}(0, T ; \mathcal{V})$,

- $\partial_{t} A_{N} \rightarrow \partial_{t} A$ weakly in $L_{r}^{2}(Q)$.

In particular, because of Lemma A.1 again, this and (4.4) imply that $\left.A(0)\right|_{\Omega_{0}}=$ $\lim _{N \rightarrow \infty}\left[\left.A_{N}(0)\right|_{\Omega_{0}}\right]=A^{0}$, where the limit is weak in $L_{r}^{2}\left(\Omega_{0}\right)$, so that $A$ satisfies the initial condition from Problem 1.

Next, we take any fixed $i \in \mathbb{N}$ so that for $N \geq i, \phi_{i} \in \mathcal{V}_{N}$. Then, we proceed as above, pass to the limit in (4.2) as $N \rightarrow \infty$, and obtain

$$
\int_{\Omega_{t}} \sigma\left(\partial_{t} A\right) \phi_{i} r d r d z+a\left(A, \phi_{i}\right)=\int_{\Omega_{\mathrm{S}}} J_{\mathrm{S}} \phi_{i} r d r d z \quad \text { a.e. } t \in[0, T] \quad \forall i \in \mathbb{N} .
$$

Hence, since the linear combinations of functions $\phi_{i}$ are dense in $\mathcal{V}$, we deduce the first equation in Problem 1. Finally, passing to the limit as $N \rightarrow \infty$ in estimate (4.14), we obtain (4.1).

In principle, estimate (4.1) holds only for a solution obtained by the regularization procedure used in this theorem. Thus, we cannot derive uniqueness of solution from this inequality. In what follows we prove a stability estimate valid for any solution to Problem 1, which will allow us to conclude that this is a well-posed problem.

TheOREM 4.3. Problem 1 has at most one solution $A$ and the following a priori estimate holds:

$$
\begin{aligned}
& \sup _{0 \leq t \leq T}\left[\int_{\Omega_{t}} \sigma|A(t)|^{2} r d r d z\right]^{1 / 2}+\|A\|_{L^{2}(0, T ; \mathcal{V})} \\
& \leq C\left[\left\|A^{0}\right\|_{L_{r}^{2}\left(\Omega_{0}\right)}+\left\|J_{\mathrm{S}}\right\|_{L^{2}\left(0, T ; L_{r}^{2}\left(\Omega_{\mathrm{S}}\right)\right)}\right] .
\end{aligned}
$$

Proof. The uniqueness follows immediately from (4.15). Thus, we only have to prove this estimate.

Let $A$ be a solution to Problem 1. Taking $Z=A(t)$ in the first equation of this problem, we obtain

$$
\int_{\Omega_{t}} \sigma\left[\partial_{t} A(t)\right] A(t) r d r d z+a(A(t), A(t))=\int_{\Omega_{\mathrm{S}}} J_{\mathrm{S}}(t) A(t) r d r d z \quad \text { a.e. } t \in[0, T] .
$$

Hence, from Theorem 3.1,

$$
\frac{1}{2} \frac{d}{d t} \int_{\Omega_{t}} \sigma|A(t)|^{2} r d r d z+\frac{1}{2} c(t, A(t), A(t))+a(A(t), A(t))=\int_{\Omega_{\mathrm{S}}} J_{\mathrm{S}}(t) A(t) r d r d z .
$$

Next, we use Lemma 4.1 and a Young's inequality to write

$$
\begin{aligned}
& \frac{1}{2} \frac{d}{d t} \int_{\Omega_{t}} \sigma|A(t)|^{2} r d r d z+\frac{\alpha}{4}\|A(t)\|_{\widetilde{H}_{r}^{1}(\Omega)}^{2} \\
& \quad \leq \frac{1}{\alpha}\left\|J_{\mathrm{S}}(t)\right\|_{L_{r}^{2}\left(\Omega_{\mathrm{S}}\right)}^{2}+\lambda \int_{\Omega_{t}} \sigma|A(t)|^{2} r d r d z .
\end{aligned}
$$

Therefore, by applying the Gronwall's lemma we conclude that

$$
\int_{\Omega_{t}} \sigma|A(t)|^{2} r d r d z \leq C\left[\int_{\Omega_{0}} \sigma\left|A^{0}\right|^{2} r d r d z+\int_{0}^{t}\left\|J_{\mathrm{S}}(s)\right\|_{L_{r}^{2}\left(\Omega_{\mathrm{S}}\right)}^{2} d s\right],
$$


where we have also used the initial condition of Problem 1. Finally, we integrate (4.16) in time from 0 to $\tau(0 \leq \tau \leq T)$ and use the above estimate to derive

$$
\begin{aligned}
& \frac{1}{2} \int_{\Omega_{\tau}} \sigma|A(\tau)|^{2} r d r d z+\frac{\alpha}{4} \int_{0}^{\tau}\|A(t)\|_{\widetilde{H}_{r}^{1}(\Omega)}^{2} d t \\
& \quad \leq C\left[\int_{\Omega_{0}} \sigma\left|A^{0}\right|^{2} r d r d z+\int_{0}^{T}\left\|J_{\mathrm{S}}(t)\right\|_{L_{r}^{2}\left(\Omega_{\mathrm{S}}\right)}^{2} d t\right],
\end{aligned}
$$

which clearly leads to (4.15). Thus, we conclude the proof.

The following result is a direct consequence of the two previous theorems.

Corollary 4.4. Under the assumptions of Theorem 4.2, Problem 1 has a unique solution and it satisfies the a priori estimates (4.15) and (4.1).

Remark 4.1. The hypotheses on the source current data $J_{\mathrm{S}}$ of Problem 1 , as well as the additional smoothness assumed in Theorem 4.2, correspond to what is usually expected in the EMF process leading to the transient eddy current problem we are analyzing. However, these assumptions can be relaxed. In what follows we describe briefly the differences arising with a less smooth data $J_{\mathrm{S}}$.

It is possible to consider a more general form of Problem 1, in which the source term $J_{\mathrm{S}}$ belongs not necessarily to $L^{2}\left(0, T ; L_{r}^{2}\left(\Omega_{\mathrm{S}}\right)\right)$ but to $L^{2}\left(0, T ; \mathcal{V}^{\prime}\right)$ (where $\mathcal{V}^{\prime}$ denotes the topological dual space of $\mathcal{V})$. In such a case, obviously the term $\int_{\Omega_{\mathrm{S}}} J_{\mathrm{S}} Z r d r d z$ has to be substituted by $\left\langle J_{\mathrm{S}}, Z\right\rangle$, where $\langle\cdot, \cdot \cdot\rangle$ denotes the duality pairing between $\mathcal{V}^{\prime}$ and $\mathcal{V}$. Theorem 4.3 is still valid under this weaker assumption, as long as $\left\|J_{\mathrm{S}}\right\|_{L^{2}\left(0, T ; L_{r}^{2}\left(\Omega_{\mathrm{S}}\right)\right)}$ is substituted by $\left\|J_{S}\right\|_{L^{2}\left(0, T ; \mathcal{V}^{\prime}\right)}$ in estimate (4.15), and no significant change is needed in its proof.

Theorem 4.2 remains valid under weaker assumptions on $J_{\mathrm{S}}$, too. In fact, it is enough to assume that $J_{\mathrm{S}} \in W^{1,1}\left(0, T ; \mathcal{V}^{\prime}\right)$ instead of $J_{\mathrm{S}} \in H^{1}\left(0, T ; L_{r}^{2}\left(\Omega_{\mathrm{S}}\right)\right)$. In such a case, estimate (4.1) holds true with $\left\|J_{\mathrm{S}}\right\|_{H^{1}\left(0, T ; L_{r}^{2}\left(\Omega_{\mathrm{S}}\right)\right)}$ substituted by $\left\|J_{\mathrm{S}}\right\|_{W^{1,1}\left(0, T, \mathcal{V}^{\prime}\right)}$. The proof of this theorem only needs to change the integrals in $\Omega_{\mathrm{S}}$ by duality pairings and to apply Lemma A.5 from [3] to the inequality analogous to (4.12), instead of Gronwall's lemma, to obtain the estimate analogous to (4.13).

5. Smoothness of the solution. The ultimate goal of this research is to develop numerical tools to solve approximately Problem 1. In order to obtain error estimates, it is particularly relevant to determine whether this solution satisfies additional regularity. In this section, we study the smoothness of the solution to Problem 1 and determine a compatibility condition among the initial data which is necessary and sufficient to have additional regularity.

First, we address time regularity. Notice that, in principle, the time derivative of the solution to Problem 1 is only defined in $Q$. However, the following theorem shows additional regularity of this derivative, which in particular implies that it is well defined a.e. in the whole $(0, T) \times \Omega$.

Theorem 5.1. Under the assumptions of Theorem 4.2, the solution to Problem 1 satisfies $\sqrt{t} \partial_{t} A \in L^{2}(0, T ; \mathcal{V})$. Moreover, the following estimate holds:

$$
\left\|\partial_{t} A(t)\right\|_{L_{r}^{2}\left(\Omega_{t}\right)} \leq \frac{C}{\sqrt{t}}\left[\left\|A^{0}\right\|_{\widetilde{H}_{r}^{1}\left(\Omega_{0}\right)}+\left\|J_{\mathrm{S}}\right\|_{H^{1}\left(0, T ; L_{r}^{2}\left(\Omega_{\mathrm{S}}\right)\right)}\right] \quad \text { a.e. } t \in(0, T] .
$$

Proof. We keep on using notation and partial results from the proof of Theorem 4.2. Recalling the definition of $\mathcal{M}_{i, j}(t)$ and using Theorem 3.1, we obtain

$$
\frac{d}{d t} \mathcal{M}_{i, j}(t)=\int_{\Omega_{t}} \sigma \operatorname{div} \boldsymbol{v} \phi_{j} \phi_{i} r d r d z+\int_{\Omega_{t}} \sigma \boldsymbol{v} \cdot \operatorname{grad}\left(\phi_{j} \phi_{i}\right) r d r d z .
$$


Since $\sigma, \boldsymbol{v}$, and $\operatorname{div} \boldsymbol{v}$ are essentially bounded, we have that $\mathcal{M}_{i, j} \in W^{1, \infty}((0, T))$. This, together with (4.10) and the assumption that $J_{\mathrm{S}} \in H^{1}\left(0, T ; L_{r}^{2}\left(\Omega_{\mathrm{S}}\right)\right)$, implies that $\boldsymbol{A}_{N}^{\varepsilon} \in H^{2}\left(0, T ; \mathbb{R}^{N}\right)$. Thus, we are allowed to differentiate (4.10) with respect to time, which leads to

$$
\sum_{j=1}^{N}\left[\mathcal{M}_{i, j}(t)+\varepsilon \mathcal{N}_{i, j}\right] \frac{d^{2}}{d t^{2}} A_{j N}^{\varepsilon}(t)+\sum_{j=1}^{N}\left[\frac{d}{d t} \mathcal{M}_{i, j}(t)+\mathcal{K}_{i, j}\right] \frac{d}{d t} A_{j N}^{\varepsilon}(t)=\frac{d}{d t} F_{i N}(t) .
$$

Next, we multiply the above equation by $t \partial_{t} A_{i N}^{\varepsilon}(t)$ and sum up from $i=1$ to $N$. Thus, we obtain

$$
\begin{aligned}
& \int_{\Omega_{t}} t \sigma\left(\partial_{t t} A_{N}^{\varepsilon}\right)\left(\partial_{t} A_{N}^{\varepsilon}\right) r d r d z+\varepsilon \int_{\Omega} t\left(\partial_{t t} A_{N}^{\varepsilon}\right)\left(\partial_{t} A_{N}^{\varepsilon}\right) r d r d z+t a\left(\partial_{t} A_{N}^{\varepsilon}, \partial_{t} A_{N}^{\varepsilon}\right) \\
& \quad+\int_{\Omega_{t}} t \sigma \operatorname{div} \boldsymbol{v}\left|\partial_{t} A_{N}^{\varepsilon}\right|^{2} r d r d z+\int_{\Omega_{t}} t \sigma \boldsymbol{v} \cdot \operatorname{grad}\left(\left|\partial_{t} A_{N}^{\varepsilon}\right|^{2}\right) r d r d z \\
& =\int_{\Omega_{\mathrm{S}}} t\left(\partial_{t} J_{\mathrm{S}}\right)\left(\partial_{t} A_{N}^{\varepsilon}\right) r d r d z
\end{aligned}
$$

From Theorem 3.1 we have

$$
\begin{aligned}
\frac{d}{d t} \int_{\Omega_{t}} t \sigma\left|\partial_{t} A_{N}^{\varepsilon}\right|^{2} r d r d z= & 2 \int_{\Omega_{t}} t \sigma\left(\partial_{t t} A_{N}^{\varepsilon}\right)\left(\partial_{t} A_{N}^{\varepsilon}\right) r d r d z \\
& +\int_{\Omega_{t}} \sigma\left|\partial_{t} A_{N}^{\varepsilon}\right|^{2} r d r d z-t c\left(t, \partial_{t} A_{N}^{\varepsilon}, \partial_{t} A_{N}^{\varepsilon}\right) .
\end{aligned}
$$

This, together with the previous equation, yields

$$
\begin{aligned}
\frac{1}{2} & \frac{d}{d t} \int_{\Omega_{t}} t \sigma\left|\partial_{t} A_{N}^{\varepsilon}\right|^{2} r d r d z+\frac{\varepsilon}{2} \frac{d}{d t} \int_{\Omega} t\left|\partial_{t} A_{N}^{\varepsilon}\right|^{2} r d r d z \\
& +\operatorname{ta}\left(\partial_{t} A_{N}^{\varepsilon}, \partial_{t} A_{N}^{\varepsilon}\right)-\frac{t}{2} c\left(t, \partial_{t} A_{N}^{\varepsilon}, \partial_{t} A_{N}^{\varepsilon}\right) \\
= & \frac{1}{2} \int_{\Omega_{t}} \sigma\left|\partial_{t} A_{N}^{\varepsilon}\right|^{2} r d r d z+\frac{\varepsilon}{2} \int_{\Omega}\left|\partial_{t} A_{N}^{\varepsilon}\right|^{2} r d r d z+\int_{\Omega_{\mathrm{S}}} t\left(\partial_{t} J_{\mathrm{S}}\right)\left(\partial_{t} A_{N}^{\varepsilon}\right) r d r d z
\end{aligned}
$$

Now, we repeat some of the arguments used in the proof of Theorem 4.3 to obtain

$$
\begin{aligned}
& \frac{1}{2} \int_{\Omega_{\tau}} \tau \sigma\left|\partial_{t} A_{N}^{\varepsilon}(\tau)\right|^{2} r d r d z+\int_{0}^{\tau} t\left\|\partial_{t} A_{N}^{\varepsilon}(t)\right\|_{\widetilde{H}_{r}^{1}(\Omega)}^{2} d t \\
& \leq C\left[\int_{0}^{\tau} \int_{\Omega_{t}} \sigma\left|\partial_{t} A_{N}^{\varepsilon}(t)\right|^{2} r d r d z d t+\varepsilon \int_{0}^{\tau} \int_{\Omega}\left|\partial_{t} A_{N}^{\varepsilon}(t)\right|^{2} r d r d z d t\right. \\
& \left.\quad+\int_{0}^{\tau} t\left\|\partial_{t} J_{\mathrm{S}}(t)\right\|_{L_{r}^{2}\left(\Omega_{\mathrm{S}}\right)}^{2} d t\right] \quad \forall \tau \in[0, T] .
\end{aligned}
$$

This estimate for $\tau=T$ together with (4.13) implies that $\sqrt{t} \partial_{t} A_{N}^{\varepsilon}$ is bounded in $L^{2}(0, T ; \mathcal{V})$. Thus, working along the same lines as in the proof of Theorem 4.2 , we 
first deduce, for fixed $N$, that $\sqrt{t} \partial_{t} A_{N}^{\varepsilon}$ converges weakly in $L^{2}(0, T ; \mathcal{V})$ as $\varepsilon \rightarrow 0+$ to a function that turns out to be $\sqrt{t} \partial_{t} A_{N}$. Next, owing to [4, Proposition III.5], $\sqrt{t} \partial_{t} A_{N}$ is also bounded in $L^{2}(0, T ; \mathcal{V})$. From this we conclude analogously that $\sqrt{t} \partial_{t} A \in$ $L^{2}(0, T ; \mathcal{V})$ (and also the weak convergence $\sqrt{t} \partial_{t} A_{N} \rightarrow \sqrt{t} \partial_{t} A$ in $L^{2}(0, T ; \mathcal{V})$ ).

On the other hand, we also have from the above inequality that $\chi_{Q}(t) \sqrt{t} \partial_{t} A_{N}^{\varepsilon}$ is bounded in $L^{\infty}\left(0, T ; L_{r}^{2}(\Omega)\right)$, where $\chi_{Q}$ stands for the characteristic function of $Q$. Hence, repeating the same steps as above, we obtain that $\chi_{Q}(t) \sqrt{t} \partial_{t} A$ is also bounded in this space, which allows us to obtain the estimate of the theorem. Thus we conclude the proof.

According to this theorem, under the sole assumptions of Theorem 4.2, the solution to Problem 1 satisfies $A \in \mathcal{C}((0, T] ; \mathcal{V})$. However, none of the above theorems yield some kind of continuity for the mapping $t \in[0, T] \mapsto A(t) \in \widetilde{H}_{r}^{1}(\Omega)$ at $t=0$. In what follows we address this issue. We begin with the following auxiliary result.

Lemma 5.2. Let $Z \in \mathcal{C}((0, T] ; \mathcal{V}) \cap L^{\infty}(0, T ; \mathcal{V})$ be such that $\partial_{t} Z \in L_{r}^{2}(Q)$. Let $\left\{t_{n}\right\}$ be a sequence such that $t_{n} \rightarrow 0+$. If $Z\left(t_{n}\right) \rightarrow \widehat{Z}$ weakly in $\mathcal{V}$, then $\left.\widehat{Z}\right|_{\Omega_{0}}=\gamma_{0} Z$, where $\gamma_{0} Z$ denotes the trace of $Z$ on $\{0\} \times \Omega_{0}$.

Proof. First notice that since $Z \in L^{\infty}(0, T ; \mathcal{V})$ and $\partial_{t} Z \in L_{r}^{2}(Q)$, it follows that $Z \in H_{r}^{1}(Q)$. Thus, according to Lemma A.1, $\gamma_{0} Z$ is well defined.

Now, let $\omega$ be an open set such that $\bar{\omega} \subset \Omega_{0}$. Because of assumptions (i)-(iv) on the motion of $\Omega_{t}$, there exists $\tau>0$ such that $(0, \tau) \times \omega \subset Q$. Therefore, $Z \in$ $H_{r}^{1}((0, \tau) \times \omega) \subset \mathcal{C}\left([0, \tau] ; L_{r}^{2}(\omega)\right)$ so that $\left.\left.Z\left(t_{n}\right)\right|_{\omega} \rightarrow\left(\gamma_{0} Z\right)\right|_{\omega}$ in $L_{r}^{2}(\omega)$.

On the other hand, since $Z\left(t_{n}\right) \rightarrow \widehat{Z}$ weakly in $\mathcal{V}$, we have that $\left.\left.Z\left(t_{n}\right)\right|_{\omega} \rightarrow \widehat{Z}\right|_{\omega}$ weakly in $L_{r}^{2}(\omega)$. Hence, $\left.\widehat{Z}\right|_{\omega}=\left.\left(\gamma_{0} Z\right)\right|_{\omega}$. Since this holds for any open set $\omega$ with $\bar{\omega} \subset \Omega_{0}$, we conclude the proof.

Our next step is to prove that without further assumptions, $\lim _{t \rightarrow 0+} A(t)$ exists weakly in $\widetilde{H}_{r}^{1}(\Omega)$. In the following theorem we show that this weak limit is completely determined by the initial data of Problem 1, namely, $A^{0}$ and the source current at the initial time $J_{\mathrm{S}}(0)$. In particular, it coincides with $A^{0}$ in $\Omega_{0}$ and with the solution of an auxiliary Dirichlet problem in $\Omega^{\mathrm{e}}:=\Omega \backslash \bar{\Omega}_{0}$.

More precisely, let $\mathcal{V}^{\mathrm{e}}:=\left\{\left.Z\right|_{\Omega^{\mathrm{e}}}: Z \in \mathcal{V}\right\}$ and $\mathcal{V}_{0}^{\mathrm{e}}:=\left\{Z \in \mathcal{V}^{\mathrm{e}}:\left.Z\right|_{\Gamma_{\mathrm{e}}}=0\right\}$, where $\Gamma_{\mathrm{e}}:=\partial \Omega^{\mathrm{e}} \cap \partial \Omega_{0}$. Let $A^{\mathrm{e}}$ be the solution of the following well-posed elliptic problem with nonhomogeneous Dirichlet boundary data: Find $A^{\mathrm{e}} \in \mathcal{V}^{\mathrm{e}}$ such that $A^{\mathrm{e}}{ }_{\Gamma_{\mathrm{e}}}=\left.A^{0}\right|_{\Gamma_{\mathrm{e}}}$ and

$$
\int_{\Omega^{\mathrm{e}}} \frac{1}{\mu} \operatorname{curl}\left(A^{\mathrm{e}} \boldsymbol{e}_{\theta}\right) \cdot \operatorname{curl}\left(Z \boldsymbol{e}_{\theta}\right) r d r d z=\int_{\Omega_{\mathrm{S}}} J_{\mathrm{S}}(0) Z r d r d z \quad \forall Z \in \mathcal{V}_{0}^{\mathrm{e}}
$$

Finally, let $\tilde{A}^{0} \in \mathcal{V}$ be the following extension of $A^{0}$ to the whole $\Omega$ :

$$
\tilde{A}^{0}:= \begin{cases}A^{0} & \text { in } \Omega_{0}, \\ A^{\mathrm{e}} & \text { in } \Omega^{\mathrm{e}} .\end{cases}
$$

We have the following result.

Theorem 5.3. Under the assumptions of Theorem 4.2, if $A$ is the solution to Problem 1, then $A(t) \rightarrow \tilde{A}^{0}$ weakly in $\mathcal{V}$ as $t \rightarrow 0+$.

Proof. First, notice that $A \in \mathcal{C}((0, T] ; \mathcal{V}) \cap L^{\infty}(0, T ; \mathcal{V})$ (cf. Theorems 5.1 and 4.2). Thus, for any sequence $\left\{t_{n}\right\}$ such that $t_{n} \rightarrow 0+$, there exists a subsequence $\left\{t_{n_{k}}\right\}$ and $\widehat{A} \in \mathcal{V}$ such that $A\left(t_{n_{k}}\right) \rightarrow \widehat{A}$ weakly in $\mathcal{V}$. Owing to Lemma $5.2,\left.\widehat{A}\right|_{\Omega_{0}}=\gamma_{0} A=A^{0}$. 
The next step is to prove that $\left.\widehat{A}\right|_{\Omega^{\mathrm{e}}}=A^{\mathrm{e}}$. From assumptions (i)-(iv) on the motion of $\Omega_{t}$, we know that for all $Z \in \mathcal{D}\left(\Omega^{\mathrm{e}}\right)$, there exists $\tau>0$ such that $\operatorname{supp}(Z) \cap \Omega_{t}=\emptyset$ for all $t \in(0, \tau)$. By taking any such $Z$ as a test function in Problem 1, we obtain

$$
\int_{\Omega^{\mathrm{e}}} \frac{1}{\mu} \operatorname{curl}\left(A(t) \boldsymbol{e}_{\theta}\right) \cdot \operatorname{curl}\left(Z \boldsymbol{e}_{\theta}\right) r d r d z=\int_{\Omega_{\mathrm{S}}} J_{\mathrm{S}}(t) Z r d r d z \quad \forall t \in(0, \tau) .
$$

Taking $t=t_{n_{k}}$, passing to the limit (recall that $J_{\mathrm{S}} \in H^{1}\left(0, T ; L_{r}^{2}\left(\Omega_{\mathrm{S}}\right)\right)$ ), and using the density of $\mathcal{D}\left(\Omega^{\mathrm{e}}\right)$ in $\mathcal{V}_{0}^{\mathrm{e}}$ (which, in turn, follows by adapting the arguments from the proofs of [11, Theorem 4.3] and [9, Lemma 3.1]), we obtain that $\widehat{A}$ satisfies (5.2). On the other hand, since $\widehat{A} \in \widetilde{H}_{r}^{1}(\Omega)$ and we have already proved that $\left.\widehat{A}\right|_{\Omega_{0}}=A^{0}$, we obtain $\left.\widehat{A}\right|_{\Gamma_{\mathrm{e}}}=\left.A^{0}\right|_{\Gamma_{\mathrm{e}}}$, so that $\left.\widehat{A}\right|_{\Omega^{\mathrm{e}}}=A^{\mathrm{e}}$.

Therefore, $\widehat{A}=\tilde{A}^{0}$ in the whole $\Omega$. Since this limit is independent of the particular sequence $\left\{t_{n}\right\}$ and of the subsequence $\left\{t_{n_{k}}\right\}$, standard arguments allow us to prove that $A(t) \rightarrow \tilde{A}^{0}$ weakly in $\mathcal{V}$, as $t \rightarrow 0+$. Thus we conclude the proof.

Next, we investigate the space regularity of the solution to Problem 1. We begin with the following immediate result.

Remark 5.1. Under the assumptions of Theorem 4.2, the unique solution to Problem 1 satisfies additional space regularity. In fact, for almost all $t \in[0, T]$, the solution $A(t)$ of this problem can be seen as the solution of an elliptic problem in the space $\mathcal{V}$, with bilinear form $a(\cdot, \cdot)$ and right-hand side $\widetilde{f}(t):=\chi_{\Omega_{\mathrm{S}}} J_{\mathrm{S}}(t)-\chi_{Q}(t) \sigma(t) \partial_{t} A(t)$. By virtue of Theorem 4.2, $\widetilde{f} \in L^{2}\left(0, T ; L_{r}^{2}(\Omega)\right)$; hence, we can apply [9, Theorem 4.1] to conclude that $A \in L^{2}\left(0, T ; \widetilde{H}_{r}^{2}(\Omega)\right)$.

A similar argument, now taking into account the estimate from Theorem 5.1, allows us to show that $A \in L^{\infty}\left(\varepsilon, T ; \widetilde{H}_{r}^{2}(\Omega)\right)$ for $\varepsilon>0$. Moreover, from the same theorem, we have that $A \in H^{1}(\varepsilon, T ; \mathcal{V})$ for $\varepsilon>0$, too. However, for numerical purposes, it is important to have $A \in H^{1}(0, T ; \mathcal{V}) \cap L^{\infty}\left(0, T ; \widetilde{H}_{r}^{2}(\Omega)\right)$.

In what follows we derive conditions on the data of the problem yielding such result. First, we establish that a necessary condition is that $\tilde{A}^{0}$ be smoother, namely, $\tilde{A}^{0} \in \widetilde{H}_{r}^{2}(\Omega)$. Notice that this is equivalent to a compatibility constraint among the initial data $A^{0}$ and $J_{\mathrm{S}}(0)$, plus additional regularity of $A^{0}$ in $\Omega_{0}$.

TheOREM 5.4. Under the assumptions of Theorem 4.2, if the solution to Problem 1 satisfies $A \in H^{1}(0, T ; \mathcal{V}) \cap L^{\infty}\left(0, T ; \widetilde{H}_{r}^{2}(\Omega)\right)$, then $\tilde{A}^{0} \in \widetilde{H}_{r}^{2}(\Omega)$.

Proof. If $A \in H^{1}(0, T ; \mathcal{V}) \cap L^{\infty}\left(0, T ; \widetilde{H}_{r}^{2}(\Omega)\right)$, then $A \in \mathcal{C}([0, T] ; \mathcal{V})$. Hence, thanks to [14, Lemma III.1.4], $A$ is weakly continuous from $[0, T]$ into $\widetilde{H}_{r}^{2}(\Omega) \cap \mathcal{V}$ and, then, $A(0) \in \widetilde{H}_{r}^{2}(\Omega) \cap \mathcal{V}$. Since $A(0)=\widetilde{A}^{0}$ (cf. Theorem 5.3), we conclude the proof.

It is natural to wonder whether the above condition is sufficient for the solution to enjoy the regularity $A \in H^{1}(0, T ; \mathcal{V}) \cap L^{\infty}\left(0, T ; \widetilde{H}_{r}^{2}(\Omega)\right)$. The next theorem gives an affirmative answer to this question.

THEOREM 5.5. Under the assumptions of Theorem 4.2, if the initial data $A^{0}$ and $J_{\mathrm{S}}(0)$ are such that $\tilde{A}^{0} \in \widetilde{H}_{r}^{2}(\Omega)$, then the solution to Problem 1 satisfies $A \in$ $H^{1}(0, T ; \mathcal{V}) \cap L^{\infty}\left(0, T ; \widetilde{H}_{r}^{2}(\Omega)\right)$.

Proof. We proceed as in the proofs of Theorems 4.2 and 5.1 and use notation and partial results from them. However, we apply now the Galerkin method with a "basis" $\left\{\phi_{n}\right\}_{n \in \mathbb{N}}$ of $\mathcal{V}$ such that $\phi_{1}=\tilde{A}^{0}$. This allows us to take $A_{N}^{0}=\tilde{A}^{0}$ for all $N \in \mathbb{N}$, since $\tilde{A}^{0} \in \widetilde{H}_{r}^{2}(\Omega) \cap \mathcal{V}$ is an extension of $A^{0}$. Note that the initial condition (4.9) of the "approximate" parabolic problem reduces to $A_{N}^{\varepsilon}(0)=\tilde{A}^{0}$; hence, it is exact and independent of both $N$ and $\varepsilon$. 
Once we arrive at (5.1) in the proof of Theorem 5.1, we multiply this equation by $\partial_{t} A_{i N}^{\varepsilon}(t)$ (instead of $t \partial_{t} A_{i N}^{\varepsilon}(t)$ ). Following essentially the same lines of that proof and using Lemma 4.1 , it is easy to obtain

$$
\begin{aligned}
& \frac{1}{2} \frac{d}{d t} \int_{\Omega_{t}} \sigma\left|\partial_{t} A_{N}^{\varepsilon}\right|^{2} r d r d z+\frac{\varepsilon}{2} \frac{d}{d t} \int_{\Omega}\left|\partial_{t} A_{N}^{\varepsilon}\right|^{2} r d r d z+\frac{\alpha}{4}\left\|\partial_{t} A_{N}^{\varepsilon}(t)\right\|_{\widetilde{H}_{r}^{1}(\Omega)}^{2} \\
& \quad \leq \lambda \int_{\Omega_{t}} \sigma\left|\partial_{t} A_{N}^{\varepsilon}(t)\right|^{2} r d r d z+\frac{1}{\alpha}\left\|\partial_{t} J_{\mathrm{S}}(t)\right\|_{L_{r}^{2}\left(\Omega_{\mathrm{S}}\right)}^{2} .
\end{aligned}
$$

Integrating the latter on $[0, \tau]$ yields

$$
\begin{aligned}
& \frac{1}{2} \int_{\Omega_{\tau}} \sigma\left|\partial_{t} A_{N}^{\varepsilon}(\tau)\right|^{2} r d r d z+\frac{\alpha}{4} \int_{0}^{\tau}\left\|\partial_{t} A_{N}^{\varepsilon}(t)\right\|_{\widetilde{H}_{r}^{1}(\Omega)}^{2} d t \\
& \leq \lambda \int_{0}^{\tau} \int_{\Omega_{t}} \sigma\left|\partial_{t} A_{N}^{\varepsilon}(t)\right|^{2} r d r d z d t+\frac{1}{\alpha} \int_{0}^{\tau}\left\|\partial_{t} J_{\mathrm{S}}(t)\right\|_{L_{r}^{2}\left(\Omega_{\mathrm{S}}\right)}^{2} d t \\
& \quad+\frac{1}{2} \int_{\Omega_{0}} \sigma\left|\partial_{t} A_{N}^{\varepsilon}(0)\right|^{2} r d r d z+\frac{\varepsilon}{2} \int_{\Omega} \sigma\left|\partial_{t} A_{N}^{\varepsilon}(0)\right|^{2} r d r d z \quad \forall \tau \in[0, T]
\end{aligned}
$$

which, taking into account (4.13), leads to

$$
\begin{aligned}
& \frac{1}{2} \int_{\Omega_{\tau}} \sigma\left|\partial_{t} A_{N}^{\varepsilon}(\tau)\right|^{2} r d r d z+\frac{\alpha}{4} \int_{0}^{\tau}\left\|\partial_{t} A_{N}^{\varepsilon}(t)\right\|_{\widetilde{H}_{r}^{1}(\Omega)}^{2} d t \\
& \leq C\left[\left\|A^{0}\right\|_{\widetilde{H}_{r}^{1}\left(\Omega_{0}\right)}^{2}+\left\|J_{\mathrm{S}}\right\|_{H^{1}\left(0, T ; L_{r}^{2}\left(\Omega_{\mathrm{S}}\right)\right)}^{2}\right] \\
& \quad+\frac{1}{2} \int_{\Omega_{0}} \sigma\left|\partial_{t} A_{N}^{\varepsilon}(0)\right|^{2} r d r d z+\frac{\varepsilon}{2} \int_{\Omega} \sigma\left|\partial_{t} A_{N}^{\varepsilon}(0)\right|^{2} r d r d z \quad \forall \tau \in[0, T] .
\end{aligned}
$$

The key point of the proof is to obtain a priori estimates for the last two integrals. With this purpose, we recall something that was shown at the beginning of the proof of Theorem 5.1: $\mathcal{M}_{i, j} \in W^{1, \infty}((0, T))$, which together with the assumption $J_{\mathrm{S}} \in$ $H^{1}\left(0, T ; L_{r}^{2}\left(\Omega_{\mathrm{S}}\right)\right)$ implies that $\boldsymbol{A}_{N}^{\varepsilon} \in H^{2}\left(0, T ; \mathbb{R}^{N}\right)$. Hence, (4.10) holds for all $t \in[0, T]$ and then the same is true for (4.8). Taking $t=0$ in this equation, multiplying by $\partial_{t} A_{i N}^{\varepsilon}(0)$, and summing up from $i=1$ to $N$, we obtain

$$
\begin{aligned}
& \int_{\Omega_{0}} \sigma\left|\partial_{t} A_{N}^{\varepsilon}(0)\right|^{2} r d r d z+\varepsilon \int_{\Omega} \sigma\left|\partial_{t} A_{N}^{\varepsilon}(0)\right|^{2} r d r d z+a\left(A_{N}^{\varepsilon}(0), \partial_{t} A_{N}^{\varepsilon}(0)\right) \\
& \quad=\int_{\Omega_{\mathrm{S}}} J_{\mathrm{S}}(0) \partial_{t} A_{N}^{\varepsilon}(0) r d r d z .
\end{aligned}
$$

Now, taking into account that $A_{N}^{\varepsilon}(0)=\tilde{A}^{0} \in \widetilde{H}_{r}^{2}(\Omega), \partial_{t} A_{N}^{\varepsilon}(0) \in \mathcal{V}$, and that $\mathcal{D}(\Omega)$ is dense in $\mathcal{V}$, we have that

$$
\begin{aligned}
a\left(A_{N}^{\varepsilon}(0), \partial_{t} A_{N}^{\varepsilon}(0)\right) & =\int_{\Omega} \frac{1}{\mu} \operatorname{curl}\left(\tilde{A}^{0} \boldsymbol{e}_{\theta}\right) \cdot \operatorname{curl}\left(\partial_{t} A_{N}^{\varepsilon}(0) \boldsymbol{e}_{\theta}\right) r d r d z \\
& =\int_{\Omega} \frac{1}{\mu} \mathcal{L}\left(\tilde{A}^{0}\right) \partial_{t} A_{N}^{\varepsilon}(0) r d r d z
\end{aligned}
$$

where

$$
\mathcal{L}\left(\tilde{A}^{0}\right):=-\frac{\partial}{\partial r}\left[\frac{1}{r} \frac{\partial\left(r \tilde{A}^{0}\right)}{\partial r}\right]-\frac{\partial^{2} \tilde{A}^{0}}{\partial z^{2}} \quad\left(=\operatorname{curl}\left[\operatorname{curl}\left(\tilde{A}^{0} \boldsymbol{e}_{\theta}\right)\right] \cdot \boldsymbol{e}_{\theta}\right) .
$$


Notice that $\mathcal{L}\left(\tilde{A}^{0}\right) \in L_{r}^{2}(\Omega)$, because of the assumption $\tilde{A}^{0} \in \widetilde{H}_{r}^{2}(\Omega)$. Moreover, the definition of $\tilde{A}^{0}$ implies that

$$
\frac{1}{\mu} \mathcal{L}\left(\tilde{A}^{0}\right)=\chi_{\Omega_{S}} J_{\mathrm{S}}(0) \quad \text { in } \Omega^{\mathrm{e}}
$$

Hence,

$\int_{\Omega} \frac{1}{\mu} \mathcal{L}\left(\tilde{A}^{0}\right) \partial_{t} A_{N}^{\varepsilon}(0) r d r d z=\int_{\Omega_{0}} \frac{1}{\mu} \mathcal{L}\left(A^{0}\right) \partial_{t} A_{N}^{\varepsilon}(0) r d r d z+\int_{\Omega_{\mathrm{S}}} J_{\mathrm{S}}(0) \partial_{t} A_{N}^{\varepsilon}(0) r d r d z$

This, together with (5.4) and (5.5), yield

$$
\int_{\Omega_{0}} \sigma\left|\partial_{t} A_{N}^{\varepsilon}(0)\right|^{2} r d r d z+\varepsilon \int_{\Omega} \sigma\left|\partial_{t} A_{N}^{\varepsilon}(0)\right|^{2} r d r d z=-\int_{\Omega_{0}} \frac{1}{\mu} \mathcal{L}\left(A^{0}\right) \partial_{t} A_{N}^{\varepsilon}(0) r d r d z
$$

which easily leads to

$$
\left\|\partial_{t} A_{N}^{\varepsilon}(0)\right\|_{L_{r}^{2}\left(\Omega_{0}\right)} \leq \frac{1}{\mu \underline{\sigma}}\left\|\mathcal{L}\left(A^{0}\right)\right\|_{L_{r}^{2}\left(\Omega_{0}\right)}
$$

and then to the a priori estimate

$$
\int_{\Omega_{0}} \sigma\left|\partial_{t} A_{N}^{\varepsilon}(0)\right|^{2} r d r d z+\varepsilon \int_{\Omega} \sigma\left|\partial_{t} A_{N}^{\varepsilon}(0)\right|^{2} r d r d z \leq \frac{1}{\mu^{2} \underline{\sigma}}\left\|\mathcal{L}\left(A^{0}\right)\right\|_{L_{r}^{2}\left(\Omega_{0}\right)}^{2} .
$$

This, together with (5.3) and the assumption that $\tilde{A}^{0} \in \widetilde{H}_{r}^{2}(\Omega)$, implies that $\partial_{t} A_{N}^{\varepsilon}$ is bounded in $L^{2}(0, T ; \mathcal{V})$ and $\chi_{Q}(t) \partial_{t} A_{N}^{\varepsilon}$ is bounded in $L^{\infty}\left(0, T ; L_{r}^{2}(\Omega)\right)$. Hence, we infer as in the final step of the proof of Theorem 5.1 that $A \in H^{1}(0, T ; \mathcal{V})$ and $\chi_{Q} \partial_{t} A \in$ $L^{\infty}\left(0, T ; L_{r}^{2}(\Omega)\right)$. Finally, the arguments in Remark 5.1 yield $A \in L^{\infty}\left(0, T ; \widetilde{H}_{r}^{2}(\Omega)\right)$ and we conclude the proof.

Remark 5.2. The compatibility condition among the initial data, $A^{0}$ and $J_{\mathrm{S}}(0)$, which ensures the assumption $\tilde{A}^{0} \in \widetilde{H}_{r}^{2}(\Omega)$ is not restrictive at all in practice. In fact, in the EMF process leading to the transient eddy current problem we have analyzed, the initial conditions are typically null: $A^{0}=0$ and $J_{\mathrm{S}}(0)=0$.

The additional regularity proved in Theorem 5.5 is typically enough to obtain error estimates for a space discretization of Problem 1 by lowest-order continuous finite elements. However, for the analysis of a full discretization, it would be useful to also have additional time regularity, for instance, $A \in H^{2}\left(0, T ; L_{r}^{2}(\Omega)\right)$. This is a subject that needs further research.

Appendix A. A trace result in $\boldsymbol{W}_{\boldsymbol{r}}^{\mathbf{1}, \boldsymbol{p}}(\boldsymbol{Q})$. As stated in Remark 3.2, the initial condition in Problem 1 makes sense because we are searching for a solution of this problem in $H_{r}^{1}(Q)$ and a trace result holds in this space. Moreover, this result was also used in the proof of Theorem 4.2.

The aim of this appendix is to prove such a trace result in $W_{r}^{1, p}(Q)$ for $p \in(1, \infty)$. We keep the notation for functional spaces in $Q$ introduced in section 3. Analogous notation will be used for functional spaces on the cylinder $(0, T) \times \widehat{\Omega}$ (with $\Omega_{t}$ replaced 
with $\widehat{\Omega})$. Moreover, we denote by $\widehat{\boldsymbol{x}}=(\widehat{r}, \widehat{z})$ a generic point in $\overline{\widehat{\Omega}}$ and by $\boldsymbol{x}=(r, z)$ a generic point in $\bar{\Omega}_{t}$.

Lemma A.1. Let $\Omega \subset\{(r, z): r>0, z \in \mathbb{R}\}$ be an open rectangle with an edge lying on $r=0$. Let $\widehat{\Omega}, \Omega_{t}, t \in[0, T]$, and $Q$ be defined as in section 3 . Let $\boldsymbol{X}$ : $[0, T] \times \overline{\widehat{\Omega}} \rightarrow \bar{\Omega}$ and $\boldsymbol{X}_{t}$ be defined as in (3.1). Suppose that assumptions (i)-(vi) hold true. Let $p \in(1, \infty)$ be fixed. Then, for all $t \in[0, T]$, there exists a unique linear continuous operator $\gamma_{t}: W_{r}^{1, p}(Q) \rightarrow L_{r}^{p}\left(\Omega_{t}\right)$ such that for all $\varphi \in \mathcal{C}^{1}(\bar{Q})$,

$$
\left(\gamma_{t} \varphi\right)(\boldsymbol{x})=\varphi(t, \boldsymbol{x}) \quad \forall \boldsymbol{x} \in \Omega_{t} .
$$

Moreover, the norm of this operator is bounded independently of $t$.

Proof. We will give a sketch of the proof, which we decompose into six steps.

Step 1. For all $p \in(1, \infty), \mathcal{C}^{1}([0, T] \times \overline{\widehat{\Omega}})$ is dense in $W_{r}^{1, p}((0, T) \times \widehat{\Omega})$.

Let $u \in W_{r}^{1, p}((0, T) \times \widehat{\Omega})$. Using standard arguments, $u$ can be approximated by finite sums of the form $\sum \psi_{i}(t) v_{i}(\widehat{\boldsymbol{x}})$, where $\psi_{i} \in \mathcal{C}^{1}([0, T])$ and $v_{i} \in W_{r}^{1, p}(\widehat{\Omega})$. Then, thanks to Theorem 4.3 from [11], each $v_{i}$ can be approximated, in the sense of $W_{r}^{1, p}(\widehat{\Omega})$, by functions in $\mathcal{C}^{1}(\bar{\Omega})$. (Here is where we use that $p \in(1, \infty)$; cf. Remark 3.3.) This yields the result.

Step 2. Let $\boldsymbol{\Psi}:[0, T] \times \overline{\widehat{\Omega}} \rightarrow[0, T] \times \bar{\Omega}$ be the mapping defined by $\boldsymbol{\Psi}(t, \widehat{\boldsymbol{x}}):=$ $(t, \boldsymbol{X}(t, \widehat{\boldsymbol{x}}))$. Then, $\boldsymbol{\Psi}:[0, T] \times \overline{\widehat{\Omega}} \rightarrow \bar{Q}$ is a homeomorphism, $\boldsymbol{\Psi} \in \mathcal{C}^{1}([0, T] \times \overline{\widehat{\Omega}})$ and $\boldsymbol{\Psi}^{-1} \in \mathcal{C}^{1}(\bar{Q})$.

The fact that $\boldsymbol{\Psi}$ is a homeomorphism onto $\bar{Q}$ follows from assumption (i) and the continuity of $\boldsymbol{X}$ by noticing that $Q=\boldsymbol{\Psi}((0, T) \times \widehat{\Omega})$. The regularity of $\boldsymbol{\Psi}$ is clear from assumption (iii), whereas that of $\boldsymbol{\Psi}^{-1}$ follows from assumptions (iii) and (iv) by using the inverse function theorem.

Step 3. Let $X_{1}$ and $X_{2}$ be the components of $\boldsymbol{X}$. There exist $c_{1}, c_{2}>0$ such that

$$
c_{1} \widehat{r} \leq X_{1}(t, \widehat{r}, \widehat{z}) \leq c_{2} \widehat{r} \quad \forall(t, \widehat{r}, \widehat{z}) \in[0, T] \times \overline{\widehat{\Omega}}
$$

First, we consider the case where $\overline{\widehat{\Omega}}$ does not intersect the axis $\widehat{r}=0$. Then, because of assumption (ii), $\boldsymbol{X}([0, T] \times \overline{\widehat{\Omega}}) \subset(0, \infty) \times \mathbb{R}$. This, together with the fact that $\boldsymbol{X}$ is continuous, implies that for all points $(t, \widehat{r}, \widehat{z})$ in the compact set $[0, T] \times \overline{\widehat{\Omega}}$, $X_{1}(t, \widehat{r}, \widehat{z})$ is bounded above and below by strictly positive constants. Since the same happens to $\widehat{r}$ for all points $(\widehat{r}, \widehat{z})$ in the compact set $\bar{\Omega}$, the property holds in this case.

Next, we consider the case where $\overline{\widehat{\Omega}}$ intersects the axis $\widehat{r}=0$. Then, according to assumption (vi), there exists $a>0$ such that for all $\delta \in(0, a), G_{\delta}:=\{(\widehat{r}, \widehat{z}) \in \overline{\widehat{\Omega}}: \widehat{r} \leq$ $\delta\}$ is a trapezoid with parallel sides aligned with the axis $\widehat{r}=0$. We write $\overline{\widehat{\Omega}}=G_{\delta} \cup F_{\delta}$ with $F_{\delta}:=\{(\widehat{r}, \widehat{z}) \in \overline{\widehat{\Omega}}: \widehat{r} \geq \delta\}$. Since $F_{\delta}$ does not intersect the axis $\widehat{r}=0$, we have just proved that (A.1) holds in $[0, T] \times F_{\delta}$. Thus, we only need to find $\delta \in(0, a)$ such that (A.1) holds in $[0, T] \times G_{\delta}$, too.

The proof of the latter follows by means of standard arguments based on the mean value theorem and the facts that $X_{1}(t, 0, \widehat{z})$ vanishes (cf. assumption (ii)), that $\frac{\partial X_{1}}{\partial \widehat{r}}$ and $\frac{\partial X_{1}}{\partial \widehat{z}}$ are bounded (cf. assumption (iii)), and that, for sufficiently small $\delta>0$, $\left|\frac{\partial X_{1}}{\partial \widetilde{z}}\right|$ is bounded in $G_{\delta}$ by a suitable small constant and $\frac{\partial X_{1}}{\partial \widetilde{r}}$ is bounded below away 
from zero. In turn, the latter can be proved by using the uniform continuity of $\frac{\partial X_{1}}{\partial \widehat{r}}$ and the following facts:

(a) $\exists \alpha>0: \operatorname{det}\left(D_{\hat{\boldsymbol{x}}} \boldsymbol{X}\right)(t, 0, \widehat{z}) \geq \alpha$ (because of assumptions (iii) and (iv));

(b) $\operatorname{det}\left(D_{\hat{\boldsymbol{x}}} \boldsymbol{X}\right)(t, 0, \widehat{z})=\frac{\partial X_{1}}{\partial \widehat{r}}(t, 0, \widehat{z}) \frac{\partial X_{2}}{\partial \widetilde{z}}(t, 0, \widehat{z})$ (because $\frac{\partial X_{1}}{\partial \widetilde{z}}(t, 0, \widehat{z})=0$ );

(c) $\frac{\partial X_{1}}{\partial \widehat{r}}(t, 0, \widehat{z}) \geq 0$ (because $X_{1}(t, \widehat{r}, \widehat{z}) \geq 0$ and $X_{1}(t, 0, \widehat{z})=0$ );

(d) $\frac{\partial X_{2}}{\partial \widetilde{z}}$ is bounded from above (because of assumption (iii)).

Step 4 . For any measurable function $u: Q \rightarrow \mathbb{R}$, let $\widehat{u}:=u \circ \boldsymbol{\Psi}:(0, T) \times \widehat{\Omega} \rightarrow \mathbb{R}$. Let $L$ be the linear operator defined by $L u:=\widehat{u}$. For all $p \in(1, \infty), L$ is an isomorphism between $L_{r}^{p}(Q)$ and $L_{r}^{p}((0, T) \times \widehat{\Omega})$ and also an isomorphism between $W_{r}^{1, p}(Q)$ and $W_{r}^{1, p}((0, T) \times \widehat{\Omega})$.

Actually, this step holds for $p \in[1, \infty)$. Using (A.1), the change of variables $(r, z)=\boldsymbol{X}(t, \widehat{r}, \widehat{z})$, and Step 2 , it is easy to prove that $u \in L_{r}^{p}(Q)$ if and only if $\widehat{u} \in L_{r}^{p}((0, T) \times \widehat{\Omega})$ and also that there exist $c_{3}, c_{4}>0$ such that

$$
c_{3}\|u\|_{L_{r}^{p}(Q)} \leq\|\widehat{u}\|_{L_{r}^{p}((0, T) \times \widehat{\Omega})} \leq c_{4}\|u\|_{L_{r}^{p}(Q)} .
$$

Hence $L: L_{r}^{p}(Q) \rightarrow L_{r}^{p}((0, T) \times \widehat{\Omega})$ is an isomorphism.

Next we prove that $L$ maps $W_{r}^{1, p}(Q)$ into $W_{r}^{1, p}((0, T) \times \widehat{\Omega})$. For $\varepsilon>0$, let $\widehat{\Omega}_{\varepsilon}:=\{(\widehat{r}, \widehat{z}) \in \widehat{\Omega}: \widehat{r}>\varepsilon\}$ and $Q_{\varepsilon}:=\boldsymbol{\Psi}\left((0, T) \times \widehat{\Omega}_{\varepsilon}\right)$. Since $u \in W_{r}^{1, p}\left(Q_{\varepsilon}\right) \simeq W^{1, p}\left(Q_{\varepsilon}\right)$, by applying the chain rule (see [4, Proposition IX.6], for instance), we have that $\widehat{u} \in W^{1, p}\left((0, T) \times \widehat{\Omega}_{\varepsilon}\right)$. Since $\varepsilon>0$ can be taken arbitrarily small, $\widehat{u} \in W_{\mathrm{loc}}^{1, p}((0, T) \times \widehat{\Omega})$ and the chain rule is valid a.e. in $(0, T) \times \widehat{\Omega}$. Using this, assumption (iii), and (A.2), we obtain that $\widehat{u} \in W_{r}^{1, p}((0, T) \times \widehat{\Omega})$ and $\|\widehat{u}\|_{W_{r}^{1, p}((0, T) \times \widehat{\Omega})} \leq C\|u\|_{W_{r}^{1, p}(Q)}$. Therefore, $L: W_{r}^{1, p}(Q) \rightarrow W_{r}^{1, p}((0, T) \times \widehat{\Omega})$ is a bounded operator. A similar argument, using now that $\Psi^{-1} \in \mathcal{C}^{1}(\bar{Q})$ (cf. Step 2), allows us to prove that $L^{-1}: W_{r}^{1, p}((0, T) \times \widehat{\Omega}) \rightarrow$ $W_{r}^{1, p}(Q)$ is also bounded.

Step 5. For all $p \in(1, \infty)$, the space $\mathcal{C}^{1}(\bar{Q})$ is dense in $W_{r}^{1, p}(Q)$.

It follows from Steps 4 and 1 and the fact that $\widehat{u} \in \mathcal{C}^{1}([0, T] \times \overline{\widehat{\Omega}})$ if and only if $u \in \mathcal{C}^{1}(\bar{Q})$, which in turn is a consequence of Step 2 .

Step 6 . Conclusion of the proof.

Let $u \in \mathcal{C}^{1}(\bar{Q})$. It is easy to show that for all $t \in[0, T]$,

$$
\begin{aligned}
& \int_{\widehat{\Omega}}|\widehat{u}(t, \widehat{r}, \widehat{z})|^{p} \widehat{r} d \widehat{r} d \widehat{z} \\
& \quad \leq \frac{2^{p-1}}{T}\left[\int_{0}^{T} \int_{\widehat{\Omega}}|\widehat{u}(\tau, \widehat{r}, \widehat{z})|^{p} \widehat{r} d \widehat{r} d \widehat{z} d \tau+T^{p} \int_{0}^{T} \int_{\widehat{\Omega}}\left|\frac{\partial \widehat{u}}{\partial t}(\tau, \widehat{r}, \widehat{z})\right|^{p} \widehat{r} d \widehat{r} d \widehat{z} d \tau\right] .
\end{aligned}
$$

Straightforward calculations using again the change of variables $(r, z)=\boldsymbol{X}(t, \widehat{r}, \widehat{z})$, (A.1), the boundedness of $\operatorname{det}\left(D_{\hat{\boldsymbol{x}}} \boldsymbol{X}\right)(t, \widehat{\boldsymbol{x}})$, the inequality above, and Step 4 lead to

$$
\int_{\Omega_{t}}|u(t, r, z)|^{p} r d r d z \leq C(T)\|u\|_{W_{r}^{1, p}(Q)}^{p} .
$$

This estimate together with Step 5 allows us to conclude the proof by means of a density argument.

Remark A.1. The above lemma still holds true under the weaker regularity assumption $\boldsymbol{X} \in \mathcal{C}^{1}([0, T] \times \overline{\widehat{\Omega}}, \bar{\Omega})$ instead of assumption (iii). 


\section{REFERENCES}

[1] A. Bermúdez, C. Reales, R. Rodríguez, and P. Salgado, Numerical analysis of a finiteelement method for the axisymmetric eddy current model of an induction furnace, IMA J. Numer. Anal., 30 (2010), pp. 654-676.

[2] A. Bermúdez, C. Reales, R. Rodríguez, and P. Salgado, Numerical analysis of a transient eddy current axisymmetric problem involving velocity terms, Numer. Methods PDEs, 28 (2012), pp. 984-1012.

[3] H. BRÉZIS, Operateurs Maximaux Monotones et Semigroups de Contractions dans les espaces de Hilbert, North-Holland, Amsterdam, 1973.

[4] H. BrÉzis, Analyse Fontionnelle, Masson, Paris, 1983.

[5] A. Buffa, Y. Maday, and F. Rapetti, Sliding mesh-mortar method for a two dimensional eddy currents model of electric engines, M2AN Math. Model. Numer. Anal., 35 (2001), pp. 191-228.

[6] R. Dautray and J.-L. Lions, Mathematical Analysis and Numerical Methods for Science and Technology, vol. 5, Springer-Verlag, Berlin, 1992.

[7] A. El-Azab, M. Garnich, And A. Kapoor, Modeling of the electromagnetic forming of sheet metals: State-of-art and future needs, J. Mater. Process. Technol., 142 (2003), pp. 744-754.

[8] J.-F. Gerbeau, C. Le Bris, And T. Lelièvre, Mathematical Methods for the Magnetohydrodynamics of Liquid Metals, Oxford University Press, Oxford, UK, 2006.

[9] J. Gopalakrishnan And J. PASCiAK, The convergence of V-cycle multigrid algorithms for axisymmetric Laplace and Maxwell equations, Math. Comp., 75 (2006), pp. 1697-1719.

[10] M.E. Gurtin, An Introduction to Continuum Mechanics, Academic Press, New York, 1981.

[11] B. Mercier AND G. RAUgel, Resolution d'un problème aux limites dans un ouvert axisymmétrique par éléments finis en $r$, $z$ et séries de Fourier en $\theta$, RAIRO Anal. Numér., 16 (1982), pp. 405-461.

[12] A. Quarteroni and A. Valli, Numerical Approximation of Partial Differential Equations, Springer-Verlag, Berlin, 1994.

[13] M. Stiemer, O. J. Unger, B. Svendsen, And H. Blum, Algorithmic formulation and numerical implementation of coupled electromagnetic-inelastic continuum models for electromagnetic metal forming, Internat. J. Numer. Methods Engrg., 68 (2006), pp. 1697-1719.

[14] R. Temam, Navier-Stokes Equations. Theory and Numerical Analysis, North-Holland, Amsterdam, 1977.

[15] M. Zlámal, Finite element solution of quasistationary nonlinear magnetic field, RAIRO Anal. Numér., 16 (1982), pp. 161-191. 